Preliminary Study of the Efficacy of Using

Nuclear Resonance Fluorescence with

Quasi-Monoenergetic Gamma-Ray

Sources for Nuclear Safeguards Assay

M. S. Johnson, D. P. McNabb, J. M. Hall, J. J. Gonzalez

May 12, 2011 
This document was prepared as an account of work sponsored by an agency of the United States government. Neither the United States government nor Lawrence Livermore National Security, LLC, nor any of their employees makes any warranty, expressed or implied, or assumes any legal liability or responsibility for the accuracy, completeness, or usefulness of any information, apparatus, product, or process disclosed, or represents that its use would not infringe privately owned rights. Reference herein to any specific commercial product, process, or service by trade name, trademark, manufacturer, or otherwise does not necessarily constitute or imply its endorsement, recommendation, or favoring by the United States government or Lawrence Livermore National Security, LLC. The views and opinions of authors expressed herein do not necessarily state or reflect those of the United States government or Lawrence Livermore National Security, LLC, and shall not be used for advertising or product endorsement purposes.

This work performed under the auspices of the U.S. Department of Energy by Lawrence Livermore National Laboratory under Contract DE-AC52-07NA27344. 


\title{
Preliminary Study of the Efficacy of Using Nuclear Resonance Fluorescence with Quasi-Monoenergetic Gamma-Ray Sources for Nuclear Safeguards Assay.
}

\author{
Micah S. Johnson (LLNL, SJSU), John J. Gonzalez (SJSU), James M. Hall (LLNL), \\ Dennis P. McNabb (LLNL). \\ LLNL (Lawrence Livermore National Laboratory, Livermore, CA) \\ SJSU (San Jose State University, Dept of Physics and Astronomy, San Jose CA)
}

\section{Executive Summary:}

We have studied the efficacy of using NRF-based techniques to assay spent nuclear fuel for Pu content using quasi-monoenergetic sources. We have developed two techniques to precisely determine the Pu content in a fuel rod/pin. One of our approaches is virtually free of systematic uncertainties. Using analytical models, we have determined the amount of time required to measure the Pu content in spent nuclear fuel rods and spent fuel assemblies to within $1 \%$ precision. We note that $\mathrm{Pu}$ content can be determined in a fuel assembly about as fast as in a single fuel pin. The performance of NRF-based assay techniques with improved photon sources, which are currently under development, will also estimated.

For follow-on research we propose to:

1. Construct research prototype detection systems for both of the NRF-based assay systems proposed in this paper and measure their calibration curves.

2. Determine the systematic errors associated with both assay methods, explore ways to reduce the errors and fold the results into future performance calculations.

3. Develop an algorithm to assay a fuel assembly.

4. Perform validation measurements using a single pin and scaled assemblies.

5. Research and develop current-mode detection and/or threshold detection techniques to improve assay times.

6. Characterize the flux of newly constructed sources and fold the results into the calculations presented here to determine the feasibility of a variety of proposed sources.

7. Collaborate with others in the safeguards community to build a prototype system and perform an NRF-based assay demonstration on spent fuel.

\section{Introduction:}

In recent conversations with our colleagues in Japan regarding the tragedy at the Fukushima Daiichi power plant, Prof. Tajima stated [Taj2011] that there is "the need for the 'toilet science' that investigates how to handle the downstream issues of energy and matter that the 'kitchen science' has spawned out has become more urgent. This of course includes our quest to 'see' the nuclear matter of relevance in much easier and more complete ways such as NRF."

There are only three nondestructive assay (NDA) systems that are in operation at nuclear power facilities to determine Pu content in spent nuclear fuel 
([Leb2001], [Kur1994], [Rin1988], and references therein). These systems do not have the capability to determine nuclear fuel burnup values (i.e. how much fuel was used/ converted via fission). Other NDA methods used by nuclear power facilities can only determine burnup values to $5 \%$ (e.g. gamma-ray emission measurements to determine average number of fissions). Burnup calculations are less accurate. Destructive assay methods offer higher precision but are too expensive to be employed at nuclear power facilities. Remote assay methods often imply incomplete assay because these methods only look at random samples rather than the entire fuel assembly.

There are many efforts that are being researched for NDA of spent nuclear fuel (c.f. [Tob2008] and references therein). One of the many methods being studied is nuclear resonance fluorescence (NRF). NRF has been studied for detection methods ([Joh2011], [Hag2009], [Haj2009], [Ber2007], [Pru2006], [Ber2005], and references therein) and has only recently been studied for assay applications. While it is clear that NRF-based techniques are capable of performing precision assay and tomography, there are real practical questions that must be addressed. First, how long (dwell time) will it take to do a measurement to the required precision? Second, what is the limit to the precision of these systems due to systematic uncertainties related to normalization and stability issues? And last, what are the costs for installing an NRF-based system?

There are several criteria that NDA methods must satisfy to be implemented at nuclear power facilities. First, new technologies must be able to determine $\mathrm{Pu}$ content with quantifiable uncertainties. Ideally, the method must be able to determine Pu content to within 1\% uncertainty (typically, the Pu content in spent fuel is about 1\%, which means that the Pu content must be known within $\pm 0.01 \%$ ). NRF is promising because the signatures are proportional to microscopic values, e.g. number of specific isotopes. This is in contrast to methods that rely on bulk values such as neutron multiplicity, gamma-ray emission, etc... Uncertainties for NRFbased methods from radiation backgrounds, scattering processes, variable material composition, and detector responses can be precisely measured and quantified. NRF has been shown to have quantifiably high confidence level for detection applications (c.f. [Pru2006],[Joh2011]). Therefore, it stands to reason that NRFbased assay will have similar benefit.

Secondly, new technologies must be compact and utilize fuel assembly cranage and other machinery-based movement apparatus already in place at the nuclear power facility. This is difficult for NRF because realistic usage of NRF techniques require an accelerator, which can be quite large. Quasi-monoenergetic Compton backscatter sources offer the best signal-to-noise ratio (c.f. [Bar2009]). Compact versions of these sources based on inverse free electron laser methods may be the best choice (c.f. [And2010]) for NRF-based assay applications. Research to increase brightness and decrease footprint is currently underway at LLNL and other facilities and laboratories. Bremsstrahlung-based accelerators are compact and probably the initial technology of choice, but the copious amount of photons (not relevant for NRF) adds to the background and increases assay times. Other 
source technologies such as Wakefield accelerators are limited in compactness because of the large laser footprint and are severely limited in brightness.

Thirdly, new technologies must have rapid scanning capabilities. The term 'rapid' here is used loosely because the constraints on scan time for assay at a nuclear power facility is much different than for detection at a shipping port. The latter is much more stringent on time because there is a clear impact on commerce. At nuclear power facilities, the spent fuel is set aside in cooling ponds for many years (e.g. 10s of years, sometimes greater than 30 years, [Ham2011]) and can be assayed at will. It must be kept in mind that there may be on the order of 1000 assemblies in a cooling pond and must be measured in a reasonable amount time, perhaps one assembly per day. High brightness gamma-ray photon sources will reduce the amount of time for NRF-based assay.

In this report we look at aspects of the first and third requirements for NRFbased NDA. Specifically, we will present and discuss the NRF-based assay methods to determine, with high precision, the Pu content in spent nuclear fuel.

\section{NRF-Based Assay Methods:}

For this effort we studied three different methods to use NRF to assay spent nuclear fuel to determine Pu content with high precision. These methods are extensions of the two methods we studied for detection of Special Nuclear material (SNM) (c.f. [Joh2011]). The methods we used in [Joh2011] were directed primarily to answering the simple question, "Is there SNM present in a given container?" The answer is clearly a 'yes' or 'no' answer with an associated confidence level (probability of false positives/negatives). The confidence level is an important input parameter needed to set the time to 'detect' a certain amount of SNM in a certain shielding configuration. The time to 'detect' determines the role NRF-based detection plays in commerce where a rate of throughput is expected.

The question for this effort, i.e. assay, that must be answered is "How much $[\mathrm{Pu}]$ material is present in a given nuclear fuel matrix?" The important quantity in assay is precision. The precision level determines the time it takes to assay a fuel matrix. Moreover, analogous to [Joh2011], the precision level provides guidance to methods that must be used for assay.

A priori, one expects the time to assay to be much longer than the time to detect a given object because assay requires counting of all isotopes in contrast to determining whether an isotope is present or not.

As a start for developing NRF-based assay method, we began by looking at the methods we used in [Joh2011]. We focus our attention initially on a single fuel $\mathrm{rod} /$ pin shown in figure 1, but we extend our study to assay capabilities of NRF for the entire assembly in the discussion section. We must establish the time to assay a small well-defined sample, e.g. a fuel pin, and then extend to more complicated systems such as fuel assemblies.

In figure 1, top, a photon beam impinges on a single fuel pin, which has been removed from the assembly in a separate staging area. Photons that are resonant with NRF excitations from the plethora of nuclei within the fuel rod are absorbed and emitted into four-pi, creating notches in the flux spectrum (assumed to be broader than the width of the NRF state, $1 \mathrm{eV}$, after thermal broadening). Other 
processes such as elastic and inelastic, not associated with NRF, scatter flux photons into four-pi as well and attenuate the beam spectrum in a more continuous fashion.

"Reflection" detectors measure the (multiple-) scattered photons and emitted NRF photons from the fuel pin directly. The reflection detection system is subject to the radiation background from the fuel since it is in direct line-of-sight of the fuel pin (for our model we used a calculated activity spectrum of 10 year old spent fuel $\sim 0.02 / \mathrm{s} / \mathrm{eV} / \mathrm{g}$ at $2.5 \mathrm{MeV}$, calculated using ORIGEN, see [Her1986]). It is important that reflection detectors are well shielded from room backgrounds not related to NRF or the sample being assayed. It is also important to use a thick absorber in front of the detector to reduce the radiation background of the sample being assayed (the dominant contributor to the background).
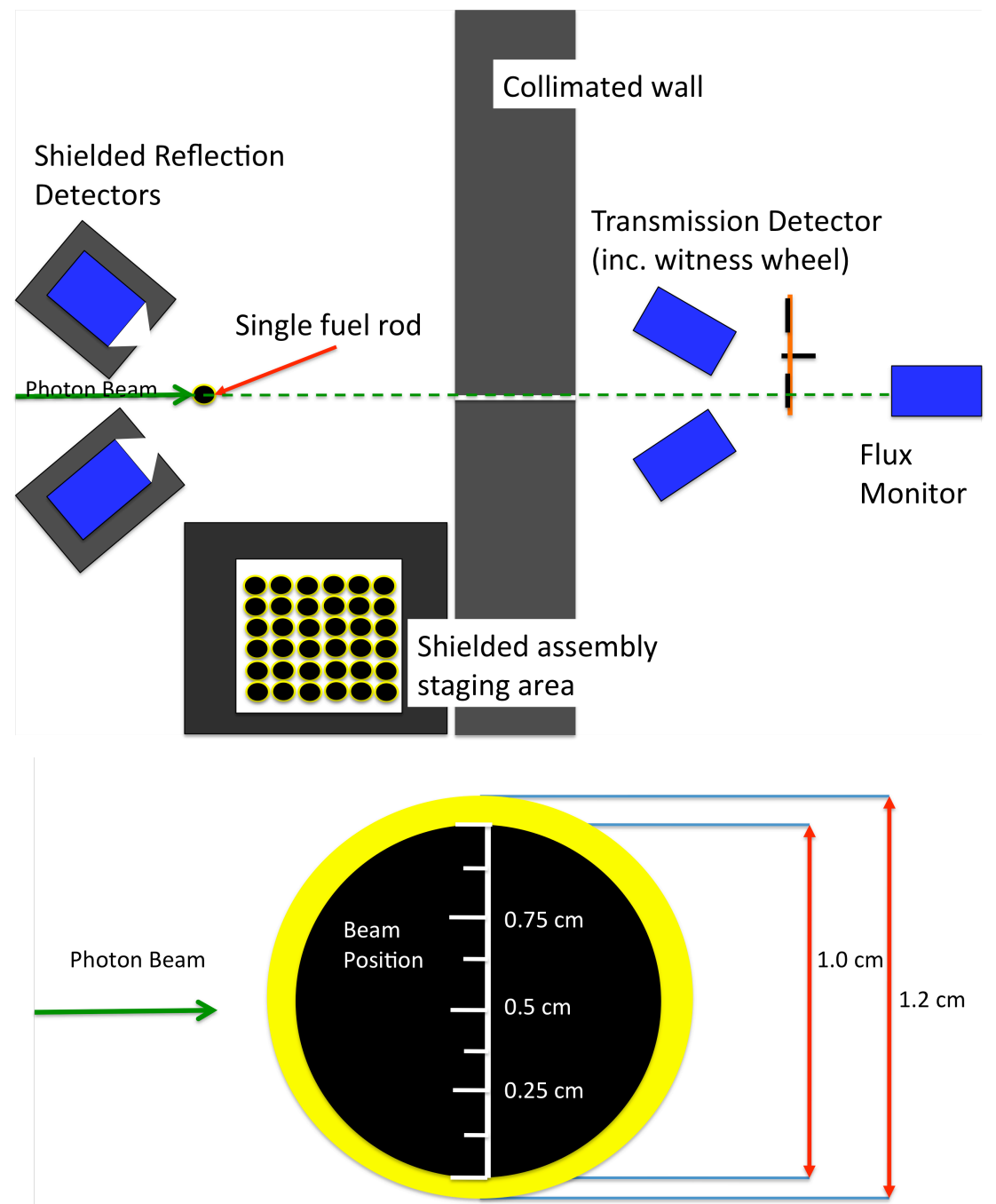

Figure 1: Schematic (upper) showing NRF-based assay method of a single nuclear fuel rod (automatically removed from an assembly). Two sets of detectors (Reflection and Transmission) measure the NRF signal (emitted and absorbed, respectively). A flux monitor is shown that will measure the attenuated beam as a fail-safe. The lower figure is a schematic of a fuel rod. The interior (black) is U02, the cladding (yellow) is $\mathrm{Zr}$. Beam position is measured from one limb of the rod to the other. See text for more details. 
The attenuated and notched beam exits the fuel rod and enters a "transmission" detector (See Ref. [Joh2011] and [Hag2009] for additional details of the transmission detector). A set of HPGe detectors are focused on a beam spot on a small sample (which can be changed out with other small samples for assaying other isotopes). The rate that the photons are scattered into the "transmission" HPGe detectors is proportional to the flux of the resonant photons. If the resonant photons are depleted from the fuel rod, then the NRF photons detected in the transmission is reduced. This is contrast to the reflection detector where more resonant material increases the reflection count rate. The background radiation from the foils is much less than the fuel material background measured by the reflection detector.

Fuel Thickness, $(\rho L)$ Fuel

As will become apparent in the discussion section, the fuel thickness, $(\rho L)$ Fuel, is an important feature that must be determined for these measurements to be valid. Variations in the fuel thickness of $\delta(\rho L)_{\text {Fuel }} /(\rho L)$ Fuel can easily be shown to add to the assay time. Therefore, precise measurements of $(\rho L)$ Fuel are just as important as measuring NRF signals. To get a precise measurement of $(\rho L)$ Fuel we propose two possible strategies.

First, we provide a thin upstream foil (e.g. copper) to measure the flux before the sample with an off-axis high-resolution (e.g. HPGe) detector. Since our quasimonoenergetic source will be around $250 \mathrm{keV}$ wide, we measure the Compton scattered flux spectrum where there are no known NRF states for the plethora of nuclei that may be present within the fuel. We then compare this integrated area to the corresponding non-Compton scattered area in the down-stream flux monitor (See Fig. 1). After a small calibration correction (for the Compton scatter), which is measured in zero-field, i.e. no fuel present, we are left with the attenuation of the fuel sample. We note that the gamma-rays above $1 \mathrm{MeV}$ are approximately attenuated the same way regardless of material. Specifically, the non-NRF absorption coefficient, $\mu_{\mathrm{A}-\mathrm{Fuel}}$, is the approximately the same whether it is virgin fuel or spent fuel. Therefore, since we know the absorption coefficient, we can use the ratio of the beam intensities to determine $(\rho L)$ Fuel. That is to say, $I / I_{0} / C_{0}=\exp \left(-\mu_{\mathrm{A}-}\right.$ Fuel $(\rho L)$ Fuel $)$, where $\mathrm{C}_{o}$ is the Compton/calibration correction, and $I_{0}$ and $I$ are the beam intensities upstream and downstream of the assayed material, respectively.

The second method we propose is to measure NRF signals upstream and downstream. The caveat is that we must use NRF material that is known to not be in the assay material. For example, we may want to use $\mathrm{C}, \mathrm{B}$, etc... These materials have large NRF strengths and therefore can be used to quickly determine the $(\rho L)$ Fuel using the same procedure as described above.

There may be other methods to determine $(\rho L)$ Fuel that may be more precise but we will leave those methods to follow-on research. Suffice it to say, although $(\rho L)_{\text {Fuel }}$ is very important information for NRF-based assay, it should be easy to determine to within a high precision. 
Detector Absorbers/Filters, $\alpha_{0}$

With large fluxes necessary to perform assay as we will see, the largest fluxrelated contribution to detector dead-time and pile-up are the Compton scatter photons from the witness foil. These photons appear at or below $300 \mathrm{keV}$ for incident energies near $2.5 \mathrm{MeV}$, where we are assuming placement of the detectors at 120-degrees in the lab frame. It is vitally important that we choose an absorber to reduce the amount of Compton photons the detector will be subjected to. We define this value as $\alpha_{0} \equiv \exp \left(-\mu_{A}(E)(\rho l)_{\text {absorber }}\right)$, where $-\mu_{A}(E)$ is the energy-dependent absorption coefficient and $(\rho \mathrm{l})_{\text {absorber }}$ is the absorber thickness. We use a simple relationship between the counting rates for NRF to the Compton rate as per the prescription given by Pruet and McNabb [PrD2006]:

$$
\frac{R_{N R F}}{R_{\text {Compton }}}=\frac{\varepsilon\left(E_{N R F}\right) \Phi_{0} N_{P u} \sigma_{N R F} \Gamma_{N R F} e^{-\mu_{A}\left(E_{N R F}\right)(\rho l)_{a b s o r b e r}}}{\varepsilon\left(E_{\text {Compton }}\right) \Phi_{0} N_{P u} \sigma_{\text {Compton }} \Gamma_{\text {Beam }} e^{-\mu_{A}\left(E_{\text {Compton }}\right)(\rho l)_{\text {absorber }}}}
$$

Using a conservative estimate in the ratio of HPGe efficiencies of $1 / 10$, the Compton cross-section for $2.5 \mathrm{MeV}$ photons $\sim 12 \mathrm{~b}$, and a beam width of $250 \mathrm{keV}$, we find that for a chosen rate of 10 times more NRF than Compton per channel, we would require the $\mathrm{Pb}$ absorber be at least $(\rho \mathrm{l})_{\text {absorber }} \sim 35 \mathrm{~g} / \mathrm{cm}^{2}$ thick (i.e. $l \sim 3 \mathrm{~cm}$ ). Subsequently, this implies that the NRF count time increases by a factor of 5 . These values are likely to be different if the detector type and efficiencies are different.

We leave $\alpha_{0}$ as a given quantity to be determined at a later time and assume that it will be known to a high precision. Moreover, we will neglect talking about backgrounds that are low in energy compared to the NRF state being used for assay. We will instead focus on backgrounds within a close range of the monoenergetic source energy. We do this for simplicity and understanding that low-energy photons can be attenuated as discussed.

It is quickly realized that straight-up photon counting in either the reflection or transmission mode have systematic uncertainties that could hamper the precision and accuracy of NRF-based assay. If we attempt to measure the amount of material present in a direct, absolute sense instead of relative to calibrated standards, the uncertainties would make dwell times impractical. To mitigate and remedy the systematic uncertainty issues, we have studied two methods to perform NRF-based assay relative to calibrated standards. These methods are called the ratio method and the fraction (or flux) method, hereafter called the $r$-method and the $f$-method, respectively.

r-method:

The $r$-method can be applied to the reflection mode or transmission mode shown in Fig. 1. The $r$-method, in either mode, determines the relative abundance of one isotope to another by taking the ratio of their respective NRF signals, e.g. $239 \mathrm{Pu} / 238 \mathrm{U}$. 
If we exclude backgrounds, signal rates go like $S \sim N \sigma \Phi \varepsilon \alpha$, where $N$ is the number of target particles, $\sigma$ is the cross-section for the interaction of the target particles and flux $(\Phi)$ photons on resonance, $\varepsilon$ is the detection efficiency (intrinsic and geometric), and $\alpha$ is the absorption factor (from a detector filter or selfattenuation in the witness foil). In reflection mode, if we solve for $N$ and take the ratio of one isotope to another, i.e. $N_{1} / N_{2}$, then we reduce the systematic uncertainties, especially if the two NRF signals are close to each other. In transmission mode, things are a bit trickier because the number of target nuclei you are looking for is in the attenuated flux because $\Phi->\Phi^{\prime}$ in the above expression for $S$.

To see how this works we explore the details. Let's define the background rates for measuring a particular isotope, $x$, as $B_{\mathrm{x}}$ (which includes elastic channels such as Delbruck and radioactive backgrounds from the target) and the overall rate as $R_{x}$. We may define the NRF counts, $S_{x}$, along with the ratio and its variance:

$$
\begin{aligned}
& \text { where } S_{x} \equiv\left(R_{x}-B_{x}\right) t \\
& \begin{array}{l}
\text { and } r \equiv \frac{S_{P_{u}}}{S_{U}} \\
\Rightarrow \sigma_{r}^{2}=\frac{1}{S_{U}^{2}} \sigma_{P u}^{2}+\left(\frac{S_{P u}}{S_{U}^{2}}\right)^{2} \sigma_{U}^{2} \\
\rightarrow \sigma_{r}^{2}=\frac{1}{\left(R_{U}-B_{U}\right)^{2} t^{2}}\left(R_{P u}+B_{P u}\right) t+\left(\frac{R_{P_{u}}-B_{P_{u}}}{\left(R_{U}-B_{U}\right)^{2} t}\right)^{2}\left(R_{U}+B_{U}\right) t=\frac{V^{2}}{t} \\
\quad \text { Equation 2 }
\end{array}
\end{aligned}
$$

where $V^{2}$ is everything in Eq. 1 except for $1 /$ t. Notice, that the first term of Eq. 2 is the dominant term for reflection because the number of NRF photons from Pu will be much less than the number of NRF photons from U (nearly two orders of magnitude difference between the number of $\mathrm{Pu}$ and $\mathrm{U}$ nuclei) subsequently reducing the contribution of the second term. The second term however, is the dominant term in transmission because the $\mathrm{NRF}$ count rates of $\mathrm{U}$ will be reduced the most from the notch created from on-resonance photons of U NRF states. Later in the paper we will discuss the different $r$-methods and refer to the leading order (LO) term and next-to leading order (NLO) term of equation 2 for the two modes, reflection and transmission.

In reflection mode, the background terms, $B$, consist of elastic and radioactive backgrounds. They are the same for $\mathrm{Pu}$ and $\mathrm{U}$, since the detectors are measuring the spent fuel. The dominant term in the background term is the radioactive background. The background rates have been calculated with ORIGEN [Her1986] to be approximately 1-10 photons/eV/s/g at $2.5 \mathrm{MeV}$ for spent fuel that has been removed from the core $\sim 10$ years.

With careful algebraic manipulation of Eq. 2 and substituting in the necessary values for $R$ one can show that $r$ and its variance in Eq. 2 can be written as polynomials in terms of $\mathrm{Pu}$ concentrations, i.e. $n$. One can also perform model simulations and fit the curve with a polynomial form as we have. We have 
determined and will demonstrate later the polynomial form is virtually linear, i.e. $r \sim m n+b$, which then leads to the variance in $r$ as a function of the Pu concentration $n:$

$$
\sigma_{r}^{2} \approx m^{2} \sigma_{n}^{2}=\frac{V^{2}}{t}
$$

Equation 3

where $m$ and $b$ are fit parameters for the ratio and their variances from the fit in Eq. 2 are assumed to measured so well that they can be neglected, $V^{2}$ is the constant of proportionality in Eq. 2 that depends on count rate, and with $T$ being the scan time. Equation 3 is explained schematically in Fig. 2. As will be shown in a later section of this paper, the ratios can be fitted to a linear function depicted in Fig. 2. The vertical axis represents the $r$-value and the horizontal axis represents the concentration $n$. If a measurement is taken of $r$ and there is an associated uncertainty then one can expect by the calibration curve that $r$ will map to $n$ and the uncertainty of $r$ will map to an uncertainty in $n$.

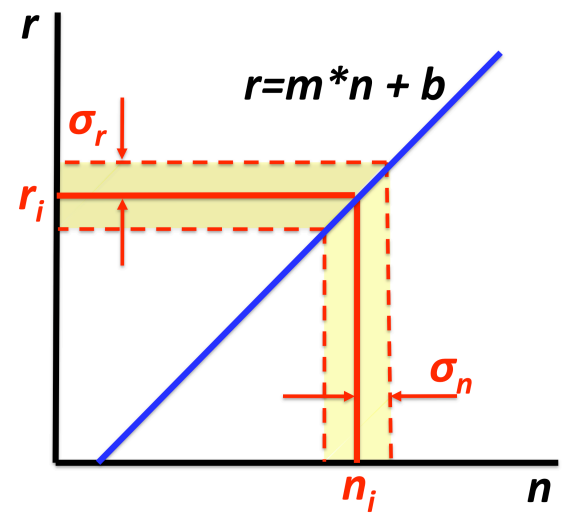

Figure 2: Schematic depicting the relationship of a measured value of $r$ to the Pu concentration $n$, in the $r$-method. Shown is an example of a measurement $r_{i}$ with an uncertainty $\sigma_{\mathrm{r}}$.

The precision, $p$, of measuring $n$ is defined $p \equiv \sigma_{\mathrm{n}} / \mathrm{n}$. It is a simple matter to determine the time for the $r$-method to be:

$$
t \approx \frac{V^{2}}{m^{2} n^{2} p^{2}}
$$

\section{f-method:}

The $f$-method can only be applied to the transmission mode. In fact, the transmission mode is modified so that an identical witness foil and set of HPGe detectors are upstream of the scanning area of Fig. 1. Important for this method is that both transmission setups are very similar and the differences are well calibrated in an open field measurement (discussed more later). The $f$-method utilizes the quantity:

$$
f_{i}=\left.\frac{\varphi^{\prime}}{\varphi}\right|_{i}
$$

Equation 5 
where $\Phi(')$ is the incident flux (attenuated flux), and $i$ is used to denote on- or off-resonance channel. The incident flux in measured with the upstream transmission detection system and the attenuated flux is measured with the downstream transmission system (See Fig. 1). The quantities $f_{i}$ are the attenuations on- and off-resonance. The attenuation is made up of nuclear and atomic processes that deplete the flux at a given energy. By taking a ratio of the $f$ quantities, we are left with the NRF contribution to the attenuation. The NRF contribution contains physical properties of the nucleus and includes the amount of resonant material. This is the value of interest. That is to say:

$$
M \equiv \frac{f_{o n}}{f_{\text {off }}}=\left(\frac{R_{o n}^{\prime}-B}{R_{o n}-B}\right)\left(\frac{R_{o f f}-B}{R_{o f f}^{\prime}-B}\right)=M_{0} e^{-\mu_{N R F}(\rho L)_{\text {Fuel }}}
$$

where $R_{i}(')$ is on- and off-resonance before (and after) the fuel, $B$ is the radiation background from the witness foil, $\mu_{N R F}$ is the attenuation coefficient associated with NRF within the fuel, $(\rho L)$ Fuel is the thickness of the fuel material being assayed, and $M_{0}$ is the calibration coefficient measured when there is no material to be assayed (effectively, $M_{0}$ is the nuclear attenuation through the upstream witness foil). The above expression in Eq. 6 can be solved for the number density of Pu nuclei in the fuel:

$$
n=-\frac{M_{\text {fuel }}}{\left.\sigma_{\text {NRF }} N_{A} \rho L\right|_{\text {fuel }}} \ln \left(\frac{M}{M_{0}}\right)
$$

where $M_{\text {fuel }}$ is the molar mass of the virgin fuel, $\sigma_{N R F}$ is the NRF cross-section of the $\mathrm{Pu}$ isotope and resonance of interest, and $N_{A}$ is Avogadro's number. Equation 7 can be written in the form: $n=c^{\prime} \ln \left(\mathrm{M} / M_{0}\right)$, where $\mathrm{c}^{\prime}$ is another calibration coefficient and is related to the material thickness being assayed and the NRF crosssection. The variance of $n$ (excluding the systematic uncertainties from $c$ ) is simply:

$$
\sigma_{n}^{2}=\frac{1}{\left(\mu_{N R F} \rho L\right)^{2}} \cdot \frac{1}{t} \cdot\left(\frac{R_{o n}^{\prime}+B}{\left(R_{o n}^{\prime}-B\right)^{2}}+\frac{R_{o n}+B}{\left(R_{o n}-B\right)^{2}}+\frac{R_{o f f}^{\prime}+B}{\left(R_{o f f}^{\prime}-B\right)^{2}}+\frac{R_{o f f}+B}{\left(R_{o f f}-B\right)^{2}}\right) \equiv \frac{1}{\left(\mu_{N R F} \rho L\right)^{2}} \cdot \frac{v^{2}}{t}
$$

where $v^{2}$ is everything in $\sigma^{2}$ (Eq. 8) except for $t$ and the coefficient, $\left(\mu_{N R F} \rho L\right)^{-2}$.

Notice what we have done, by taking ratios of simultaneous flux measurements before and after the fuel sample for on- and off-resonance channels, we will get a result whose systematic uncertainties have been nearly cancelled out. In other words, the uncertainties of the $f$-method are almost purely statistical if the calibrations do not shift substantially during a measurement. Moreover, the $f$ method yields a direct measure of the number of isotopes of interest, arguably a direct determination of the mass via $n$. This is in contrast to the $r$-method, which only yields the relative ratio of isotopes of interest.

Later in this paper we refer to the LO and NLO term of Eq. 9, which refers to the third and first term, respectively. It should be obvious that these two terms are 
the dominant terms because they represent the respective count rates in the downstream detectors, after the attenuation through the fuel sample.

The precision to measure the number density of $\mathrm{Pu}$ in the nuclear fuel is simply defined as: $p \equiv \sigma_{\mathrm{n}} / \mathrm{n}$. The time to determine the absolute concentration for the $f$-method is (after some algebra and ignoring systematic uncertainties, i.e. assumed calibrations to be stable):

$$
t_{f}=\frac{v^{2}}{p^{2}\left(\ln \left(\frac{M}{M_{0}}\right)\right)^{2}}
$$

Notice in Eq. 9 that if $M=M_{0}$, the scan time becomes infinite. This is expected because in Eq. $6 \mathrm{M}$ is proportional to the exponential of $\rho L$. This would imply there is no assay material present and would in fact take an infinite amount of time to assay nothing.

\section{Scenarios:}

As stated earlier, we have chosen in this early study to focus our efforts on a nuclear fuel rod/pin and will broaden our discussion to fuel assemblies later. We reserve more detailed studies of fuel assemblies with our newly developed GEANTNRF package (c.f. [Joh2010]) as a follow-on project. We have chosen to use an analytical model we developed from an extension of our detection model in [Joh2011] because of its ease of use and its validation through measurements.

For our simulated rod we used a standard fuel rod geometry $(1.0 \mathrm{~cm}$ diameter) with a spent fuel mixture of various quantities of $\mathrm{UO} 2, \mathrm{Pu}$, and fission products (typically spent fuel is about $96 \% \mathrm{UO}_{2}, 1 \% \mathrm{Pu}$, and $3 \%$ fission products). The density of the fuel was $10.96 \mathrm{~g} / \mathrm{cm} 3$. The fission products were obtained using fission distribution from data and calculations [Vog2010]. The breakdown of uranium isotopes includes 3\% 235U (i.e. initial enrichment (IE) is 3\%) and 97\% $238 \mathrm{U}$ and remained constant. We reserve the burn of $235 \mathrm{U}$ for a follow-on project. For this project we are only interested in Pu content and without loss of generality we keep the enrichment constant. Our model allows for any number of isotope composition of fuel or otherwise (e.g. fission products at various stages of cooling, enrichment, etc...). For our model calculations we used varying concentration of $\mathrm{Pu}$ to estimate the performance capabilities of determining Pu content with NRF (e.g. $0.0 \%, 0.25 \%, 0.50 \%, 0.75 \%$ and $1.0 \%$ ). The ratio of $239 \mathrm{Pu}$ to $240 \mathrm{Pu}$ was held constant for the calculations at 94\% 239Pu and 6\% $240 \mathrm{Pu}$.

Our model allows for independent spatial distributions of the fuel, plutonium, and fission fragments. Spatial distributions are important for studying burnup from fuel rods that come from various points within the fuel assembly and the nuclear core. The current basis for reactor performance estimates is set on uniform burnup. However, it is known that reactors do not produce uniform burns. Upgrades in burnup codes (e.g. ORIGEN) include non-uniform burns. For our calculations we used a flat spatial distribution of fuel, $\mathrm{Pu}$, and fission products (i.e. homogeneous distributions of $\mathrm{Pu}$ and fission products). 
Table 1: List of isotopes we have used in our calculations. Listed are the resonant energies and their respective integrated cross-sections. Aslo given are the relevent citations for these values.

\begin{tabular}{|l|l|l|l|}
\hline Isotope & $\begin{array}{l}\text { Resonance } \\
{[\mathrm{MeV}]}\end{array}$ & $\begin{array}{l}\text { Integrated Cross- } \\
\text { Section }[\mathrm{eV} \mathrm{b}]\end{array}$ & Reference Source \\
\hline $238 \mathrm{U}$ & 2.468 & 61 & {$[$ Hei1988] } \\
\hline $239 \mathrm{Pu}$ & 2.464 & 8 & {$[$ Ber2008] } \\
\hline $240 \mathrm{Pu}$ & 2.577 & 174 & This work (see table 3) \\
\hline $103 \mathrm{Rh}$ & 2.516 & 4286 & {$[$ Cau1981] } \\
\hline
\end{tabular}

The NRF energies and states that we have focused on for these calculations are listed in table 1. 103Rh was chosen because it is a well-known fission product (peak of the lower mass component of the fission fragment distribution) of nuclear fuel and it has a well-known yield from spent nuclear fuel (3.6\% of the fission yield). $103 \mathrm{Rh}$ was also chosen because of an NRF state at $2.516 \mathrm{MeV}$. The NRF state listed above for $240 \mathrm{Pu}$, is a preliminary result from recent measurements (See Sec. Pu NRF Measurements). For our model we assumed no initial fission product content and that the fission products grew in as a function of burn time. We assumed that the fission yield was always a factor of 3 more than the Pu production, which is consistent with spent nuclear fuel.

We used HPGe detectors for our model estimates. These detectors are important because it allows distinction between the various NRF lines that may be in close proximity, especially since the incident photons are quasi-monoenergetic. The HPGe choice is important for the $r$-method where two or more lines are compared and analyzed. The HPGe choice is also important for the $f$-method where the on-resonance signal is compared to the off-resonance channel. A modified version of the $f$-method may be a calorimetric technique where low-resolution detectors are sufficient. We will leave that for follow-on research.

For the photon source, we mimicked the High Intensity Gamma-ray Source (HIGS) at Duke University's Free Electron Laser Laboratory (DFELL). The purpose for using HIGS source was to give a realistic sense of the application capability with current technology. We chose a beam with energy of $2.5 \mathrm{MeV}$ and a bandwidth of $250 \mathrm{keV}$ and a Gaussian shape. The peak flux was set at 400 photons/eV/sec, which is higher than the usual operations at HIGS, but since our models take advantage of a broader beam, we can allow more flux at the cost of bandwidth [Yin2011]. (Our analytical model allows various photon distributions to mimic any source. For example, narrow bandwidth sources such as Compton backscatter sources have sharp cutoffs on the high-energy side and very long low-energy tails.) The values for the resultant flux on resonance are given in Tab 2.

Table 2: List of incident flux values on resonance. We have assumed an incident flux of 400/eV/sec at 2.5 $\mathrm{MeV}$ with a $250 \mathrm{keV}$ broad beam. Without loss of generality we have assumed the beam to be Gaussian.

\begin{tabular}{|l|l|l|}
\hline Isotope & $\begin{array}{l}\text { Resonance } \\
{[\mathrm{MeV}]}\end{array}$ & $\begin{array}{l}\text { Flux on resonance } \\
\text { [photons/eV/sec] }\end{array}$ \\
\hline $238 \mathrm{U}$ & 2.468 & 382 \\
\hline
\end{tabular}




\begin{tabular}{|l|l|l|}
\hline $239 \mathrm{Pu}$ & 2.464 & 378 \\
\hline $240 \mathrm{Pu}$ & 2.577 & 310 \\
\hline $103 \mathrm{Rh}$ & 2.516 & 395 \\
\hline
\end{tabular}

Another key feature to our model is the scattering processes. We consider up to secondary scattering processes including both elastic and inelastic channels. We exclude higher order scattering because they do not contribute significantly to the end result and with that level of detail the calculation effectively becomes a MC simulation. This only holds true for quasi-monoenergetic sources when compared to bremsstrahlung.

The inelastic processes include NRF, Compton scattering, photoelectric effect, and pair production. The elastic processes include Rayleigh, Thomson, and Delbruck scattering.

\section{Pu NRF Measurements:}

As part of the tasking for this effort, we have performed NRF measurements of an LLNL Pu target at the HIGS facility at DFELL. The HIGS beam was tuned to various energies between $2.1 \mathrm{MeV}$ and $2.7 \mathrm{MeV}$ and run at an average current of $10^{7}$ photons/second with about $2 \%$ bandwidth. We chose this energy interval to validate the NRF states observed in our previous measurements of Pu with a bremsstrahlung source [Ber2008]. We used a 100\% polarized beam at HIGS and situated four of our $60 \% \mathrm{HPGe}$ detectors to in-plane and out-of-plane orientations at a lab angle of 90-degrees to measure the spin and parity of NRF states (c.f.

[Pie2002]). The LLNL Pu targets are 94\% 239Pu and 6\% 240Pu. The states in [Ber2008]) were deduced to be 239Pu based on systematics.

The analysis of the data is still ongoing at the time of this report and the preliminary results are presented in Table 3 .

Table 3: Preliminary results for NRF measurements of Pu. Assignments made for this effort are based on gamma-ray asymmetry.

\begin{tabular}{|c|c|c|c|}
\hline $\begin{array}{c}\text { Tentative } \\
\text { Assignment }\end{array}$ & $\begin{array}{c}\text { Energy } \\
(\mathrm{MeV})\end{array}$ & $\begin{array}{c}\text { Integrated Cross- } \\
\text { Section }(\mathrm{eVb})\end{array}$ & $\begin{array}{c}\text { Literature Cross-Section } \\
(\mathrm{eVb})[\text { Ber2008] }\end{array}$ \\
\hline $239 \mathrm{Pu}$ & 2.135 & $7(2)$ & $4(2)$ \\
\hline $239 \mathrm{Pu}$ & 2.144 & $10(2)$ & $13(2)$ \\
\hline $239 \mathrm{Pu}$ & 2.151 & $5(1)$ & $5(2)$ \\
\hline $239 \mathrm{Pu}$ & 2.166 & $4(1)$ & na \\
\hline $239 \mathrm{Pu}$ & 2.174 & $4(1)$ & na \\
\hline $239 \mathrm{Pu}$ & 2.183 & $2(1)$ & na \\
\hline $239 \mathrm{Pu}$ & 2.424 & $13(2)$ & $9(2)$ \\
\hline $239 \mathrm{Pu}$ & 2.454 & $6(1)$ & $6(4)$ \\
\hline $239 \mathrm{Pu}$ & 2.461 & $5(1)$ & $8(4)$ \\
\hline $239 \mathrm{Pu}$ & 2.464 & $7(2)$ & $6(2)$ \\
\hline $239 \mathrm{Pu}$ & 2.471 & $6(1)$ & $9(3)\left\{\right.$ Prev. ass. to $\left.{ }^{239} \mathrm{Pu}\right\}$ \\
\hline $240 \mathrm{Pu}$ & 2.432 & $156(28)$ &
\end{tabular}




\begin{tabular}{|c|c|c|c|}
\hline $239 \mathrm{Pu}$ & 2.567 & $7(1)$ & na \\
\hline $240 \mathrm{Pu}$ & 2.577 & $174(73)$ & na \\
\hline $239 \mathrm{Pu}$ & 2.596 & $5(1)$ & na \\
\hline
\end{tabular}

The benefit of measuring the parity of the NRF states is that for odd-mass nuclei such as $239 \mathrm{Pu}$, dipole states are often fragmented among a plethora of projections of the spin state and symmetry axis. The emitted NRF photons for oddmass nuclei are emitted at various angles according to the projection angle and have no discernable asymmetry relative to the beam polarization. For even-even nuclei the dipole states all point along the symmetry axis of the nucleus and therefore can be relatively finite and discrete. Moreover, the direction of the emitted NRF photon is fixed by the nuclear orientation and beam polarization giving in-plane and out-ofplane asymmetry.

Preliminary results in Tab. 3 indicate that all of the NRF states observed in [Ber2008] are in fact present and are still assigned to 239Pu with the exception of the level at $2.432 \mathrm{MeV}$. The assignments are based on the symmetry of the in-plane and out-of-plane detectors. More detailed information regarding the measurements including the final results will be reserved for a planned publication in a peerreviewed journal.

\section{Model Estimates:}

The simulations were performed for varying concentrations of Pu and are displayed in all figures except when otherwise noted (the color array is black $=0.0 \%$, red $=0.25 \%$, green $=0.50 \%$, blue $=0.75 \%$, and orange $=1.0 \%$ ). In all plots hereafter, the horizontal axis is a measure of beam position unless otherwise noted. Beam position is the position the beam on the diameter of the fuel rod (See Fig. 1 lower). For a single detector in reflection mode, the beam position goes from the side furthest from the detector (beam position $=0.0 \mathrm{~cm}$ ) to the side nearest to the detector $($ beam position $=1.0 \mathrm{~cm}$ ). For the opposing reflection detector, the beam positions are reversed.

\section{Reflection Mode}

We begin by simulating the reflection mode detection scheme. Table 4 lists ad hoc (back-of-the-envelope) calculations for what we might expect in terms of $\mathrm{NRF}$ count rates. We use these values as a simple check of the simulations for the figures given in the far right column in Tab. 4. 
Table 4: Ad hoc estimates for NRF count rate in reflection mode for a single fuel rod with $\sim 1 \% \mathrm{Pu}$. The number of $\mathrm{UO2}$ is $2.5 \times 10^{-2}$ [nuclei/barn], we used $\alpha \varepsilon \sim 1 \times 10^{-3}$, and $\mathrm{L}=1 \mathrm{~cm}$. We used a Pu concentration of $1 \%$. These values are not expected to be exact but are used to approximate the associated figures.

\begin{tabular}{|l|l|l|l|l|l|}
\hline Isotope & $\begin{array}{l}\text { Resonance } \\
{[\mathrm{MeV}]}\end{array}$ & $\begin{array}{l}\text { Integrated } \\
\text { Cross- } \\
\text { Section } \\
{[\mathrm{eV} \mathrm{b}]}\end{array}$ & $\begin{array}{l}\text { Resonant flux } \\
\text { [photons/eV/sec] }\end{array}$ & $\begin{array}{l}\text { Ad hoc } \\
\text { estimates } \\
\text { for NRF } \\
\text { count rate } \\
\text { [cps] }\end{array}$ & $\begin{array}{l}\text { Associated } \\
\text { figure (for } \\
\text { comparison) }\end{array}$ \\
\hline $238 \mathrm{U}$ & 2.468 & 61 & 382 & $\sim 0.1$ & Fig. 3 \\
\hline $239 \mathrm{Pu}$ & 2.464 & 8 & 378 & $\sim 0.0004$ & Fig. 4 \\
\hline $240 \mathrm{Pu}$ & 2.577 & 174 & 310 & $\sim 0.0004$ & Fig. 5 \\
\hline $103 \mathrm{Rh}$ & 2.516 & 4286 & 395 & $\sim 0.02$ & Fig. 6 \\
\hline
\end{tabular}

Figure 3 is a plot of the $238 \mathrm{U}$ NRF count rate in units of counts per second (cps) in reflection mode as a function of beam position.

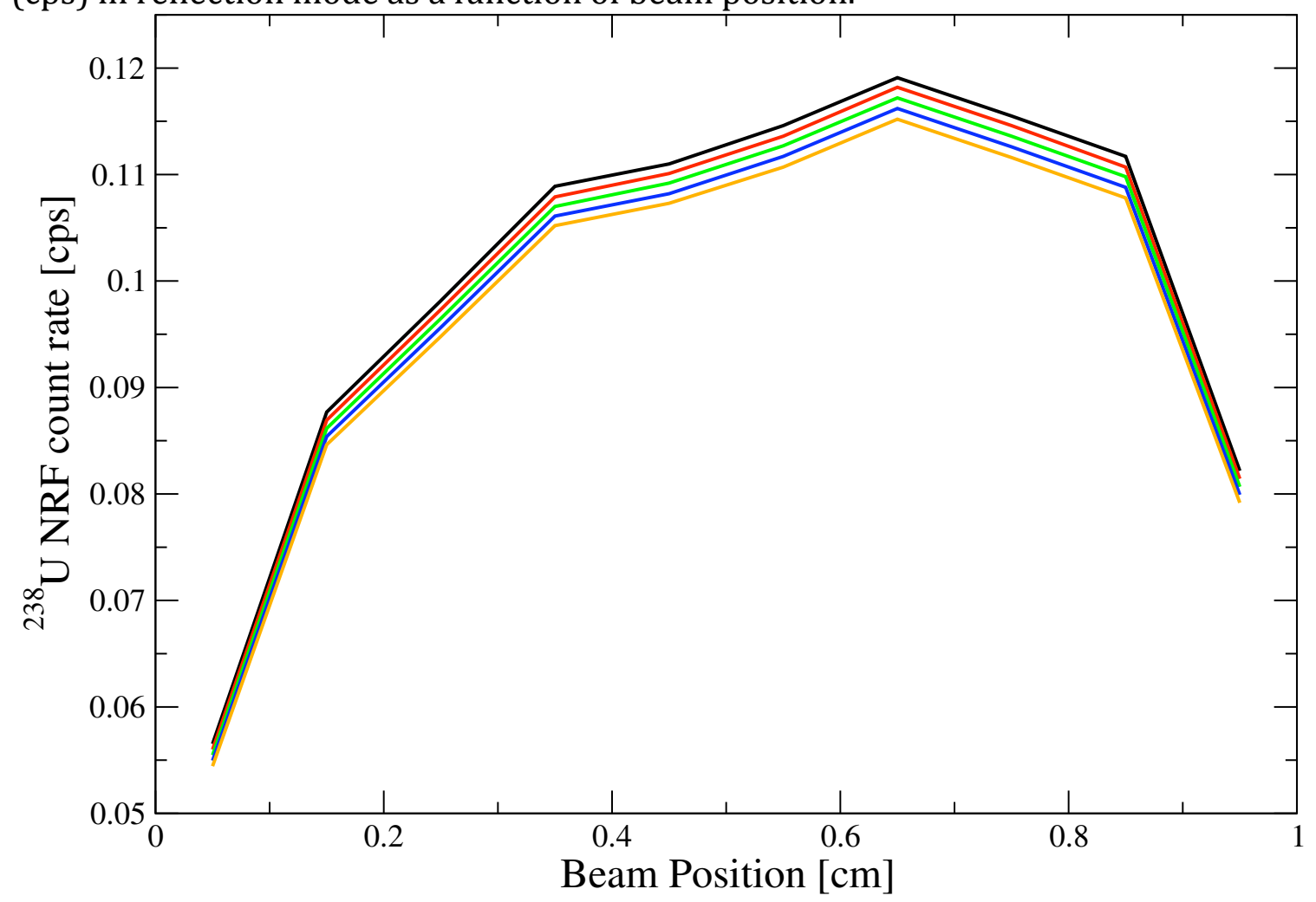

Figure 3: Plot of 238U NRF count rate of one reflection detector (nearest to beam position at $1.0 \mathrm{~cm}$ ).

The higher count rate nearest the detector is due to lower attenuation of NRF signal through the fuel rod. The different curves represent different percentages of $\mathrm{Pu}$ (i.e. black $=0.0 \%$, red $=0.25 \%$, green $=0.5 \%$, blue $=\mathbf{0 . 7 5 \%}$, orange=1.0\%). The peak flux was assumed to be $400 / \mathrm{eV} / \mathrm{s}$. See text for more details.

The increased counts nearest the detector are higher because of the lower attenuation of signals as they pass through the rod from the interaction point en route to the detector. (Clearly, the detector on the opposite side of the beam would have the reverse of this plot.) The functions plotted in Fig. 3 are of the various $\mathrm{Pu}$ concentrations (listed above). 
Figure 4 is a plot of the 239Pu NRF count rate in reflection mode as a function of beam position from the side furthest from the detector $(0 \mathrm{~cm})$ to the side nearest to the detector $(1 \mathrm{~cm})$. The trend of the counts as a function of beam position is interpreted in the same way as $238 \mathrm{U}$ in Fig. 3 above. The curves plotted in Fig. 4 are of the various Pu concentrations (listed above) and are shown in different colors from $0.0 \%$, black to $1.0 \%$ in orange. It is obvious that the more Pu in the fuel sample the greater the number of measured NRF counts will be for Pu.

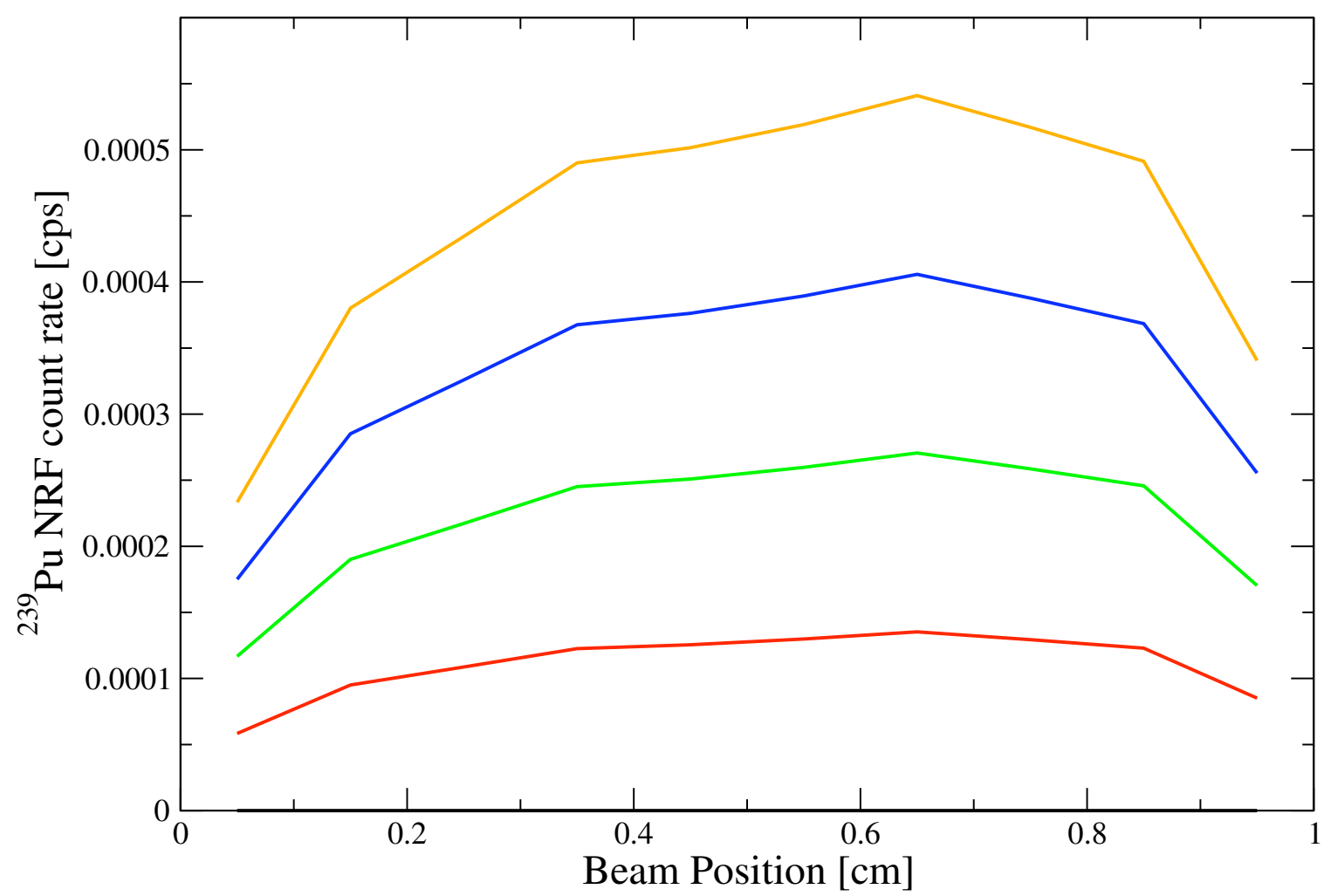

Figure 4: Plot of 239Pu NRF count rate of one reflection detector (nearest to beam position at $1.0 \mathrm{~cm}$ ). The different curves represent different percentages of $\mathrm{Pu}$ (i.e. black $=0.0 \%$, red $=0.25 \%$, green=0.5\%, blue $=0.75 \%$, orange=1.0\%). The peak flux was assumed to be $400 / \mathrm{eV} / \mathrm{s}$. See text for more details.

Figure 5 is a plot of the $240 \mathrm{Pu} \mathrm{NRF}$ count rate in reflection mode as a function of beam position from the side furthest from the detector to the side nearest to the detector. The trend of the counts as a function of beam position is interpreted in the same way as 239Pu in Fig. 4 above. The curves plotted are of the various Pu concentrations (listed above) and are shown in different colors from $0.0 \%$, black to $1.0 \%$ in orange. (Recall that $240 \mathrm{Pu}$ is only $6 \%$ of the Pu content.) It is obvious that the more Pu in the fuel sample the greater the number of measured NRF counts will be for $\mathrm{Pu}$. 


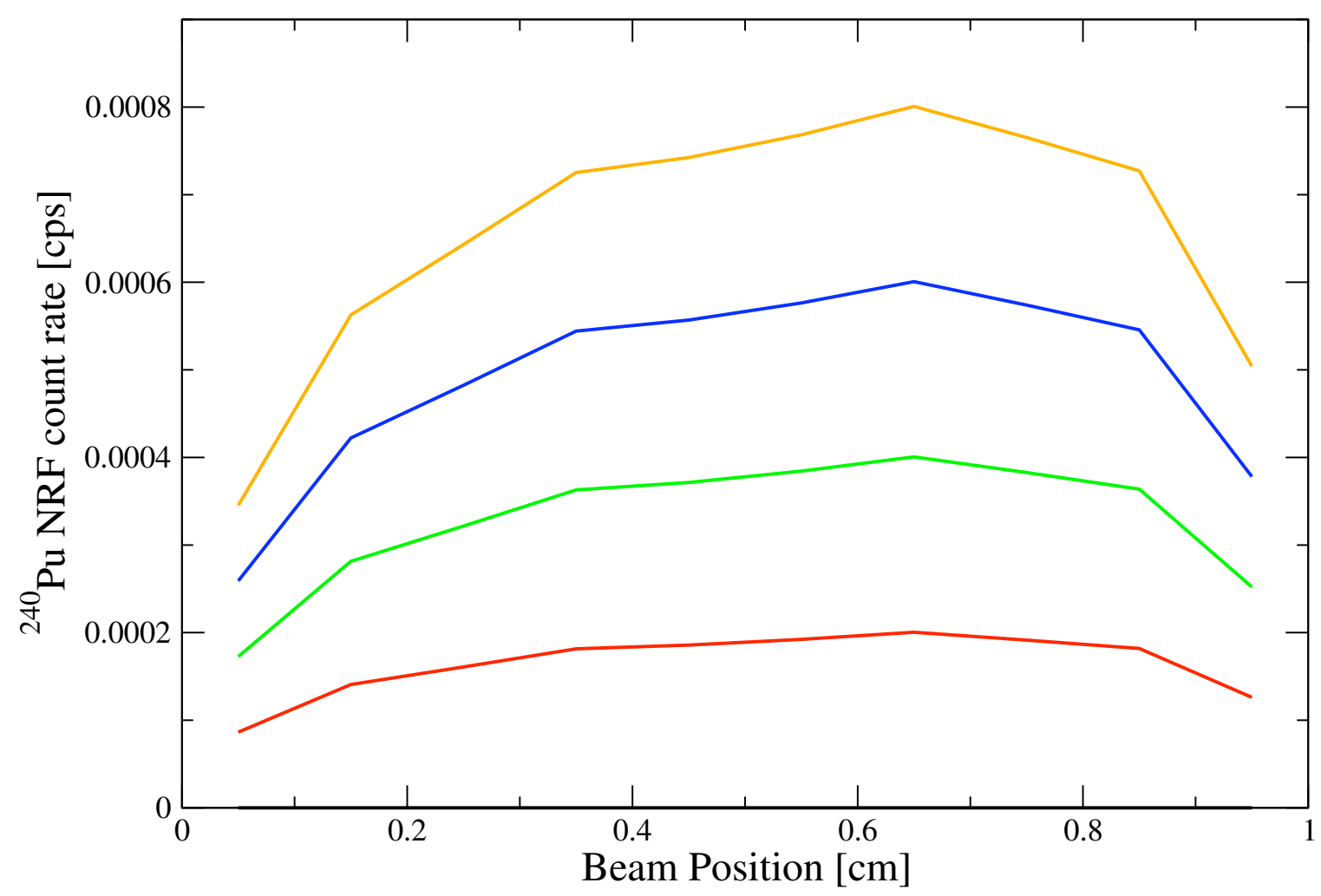

Figure 5: Plot of 240Pu NRF count rate of one reflection detector (nearest to beam position at $1.0 \mathrm{~cm}$ ). The different curves represent different percentages of Pu (i.e. black $=0.0 \%$, red $=0.25 \%$, green $=0.5 \%$, blue $=0.75 \%$, orange $=1.0 \%$ ). The peak flux was assumed to be $400 / \mathrm{eV} / \mathrm{s}$. See text for more details.

Figure 6 is a plot of the 103Rh (a dominant fission product) NRF count rate in reflection mode as a function of beam position from the side furthest from the detector to the side nearest to the detector. The trend of the counts as a function of beam position is interpreted in the same way as Pu in Figs. 4 and 5 above. The curves plotted are of the various Pu concentrations (listed above) and are shown in different colors from $0.0 \%$, black to $1.0 \%$ in orange. Equivalently, these can be read as black $0.0 \%$ fission products to $3.0 \%$ fission products in increments of $0.75 \%$. (Recall that $103 \mathrm{Rh}$ is only $3.6 \%$ of the total fission yield.) 


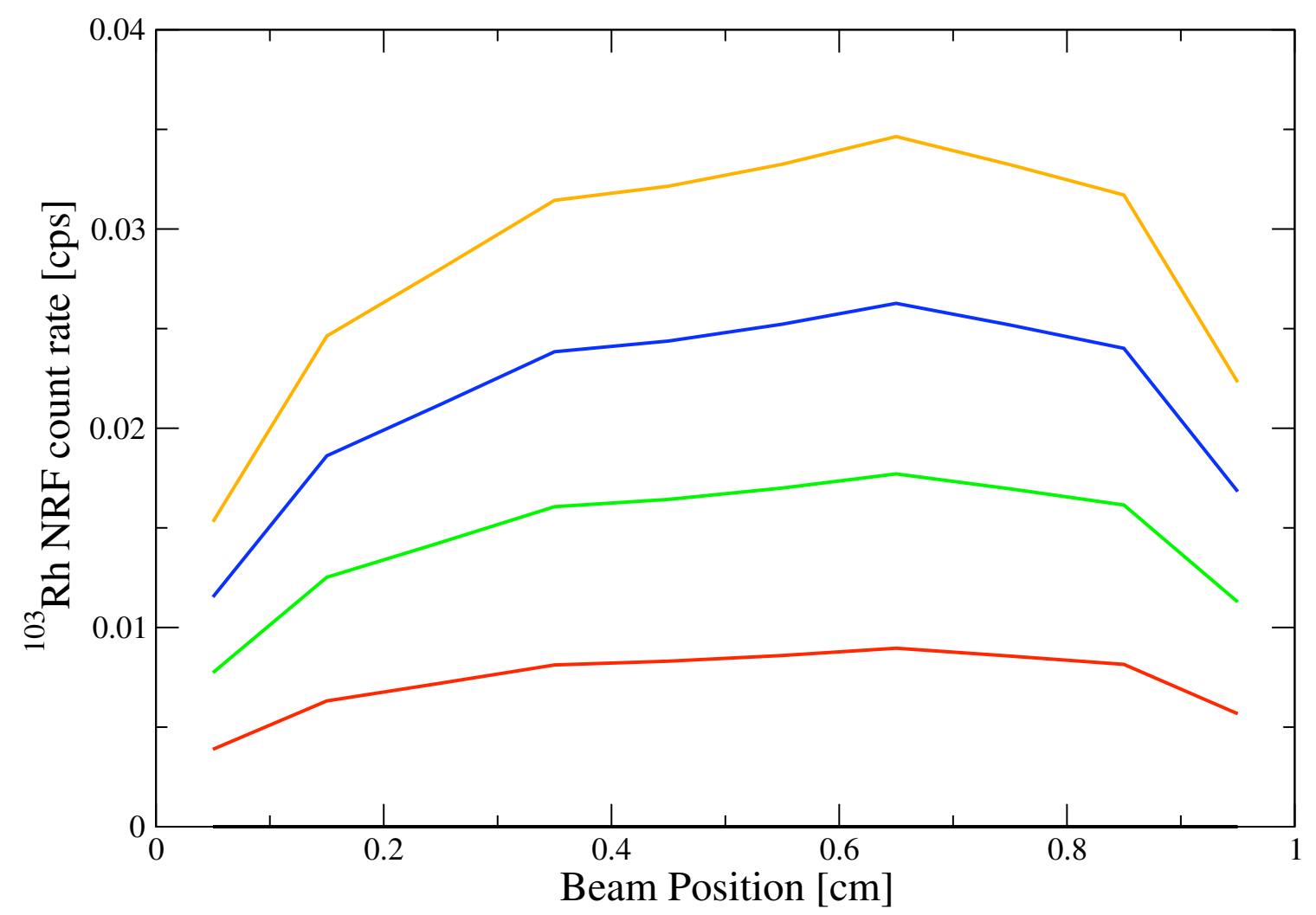

Figure 6: Plot of 103Rh (a dominant and well known fission fragment) NRF count rate of one reflection detector (nearest to beam position at $1.5 \mathrm{~cm}$ ). The different curves represent different percentages of Pu (i.e. black $=0.0 \%$, red $=0.25 \%$, green $=0.5 \%$, blue $=0.75 \%$, orange $=1.0 \%$ ). The peak flux was assumed to be $400 / \mathrm{eV} / \mathrm{s}$. See text for more details.

\section{Transmission Mode}

We now look at our simulations for the transmission mode detection scheme. Table 5 lists our ad hoc calculations for what we might expect in terms of NRF count rates in transmission mode. We use these values as a simple check of the simulations for the figures given in the far right column in Tab. 5.

Table 5: Ad hoc estimates for NRF count rate in transmission mode for a single fuel rod with $\mathbf{n} \sim 1 \%$. We used $\alpha \varepsilon \sim 0.5 \times 10^{-2}$ and a witness foil thickness of $1=3 \mathrm{~mm}$. We used a Pu concentration of $1 \%$ for the fuel rod. These values are not expected to be exact but are used to approximate the associated figures.

\begin{tabular}{|l|l|l|l|l|l|}
\hline Isotope & $\begin{array}{l}\text { Resonance } \\
{[\mathrm{MeV}]}\end{array}$ & $\begin{array}{l}\text { Integrated } \\
\text { Cross- } \\
\text { Section } \\
{[\mathrm{eV} \text { b] }}\end{array}$ & $\begin{array}{l}\text { Resonant flux } \\
\text { [photons/eV/sec }]\end{array}$ & $\begin{array}{l}\text { Ad hoc } \\
\text { estimates } \\
\text { for NRF } \\
\text { count rate } \\
{[\mathrm{cps}]}\end{array}$ & $\begin{array}{l}\text { Associated } \\
\text { figure (for } \\
\text { comparison) }\end{array}$ \\
\hline $238 \mathrm{U}$ & 2.468 & 61 & 147 & $\sim 1.1$ & Fig. 7 \\
\hline $239 \mathrm{Pu}$ & 2.464 & 8 & 230 & $\sim 0.2$ & Fig. 8 \\
\hline $240 \mathrm{Pu}$ & 2.577 & 174 & 189 & $\sim 4.0$ & Fig. 9 \\
\hline $103 \mathrm{Rh}$ & 2.516 & 4286 & 215 & $\sim 161$. & Not shown \\
\hline
\end{tabular}


Figure 7 is a plot of the $238 \mathrm{U}$ NRF count rate in transmission mode as a function of beam position. The trends of the counts as a function of beam position is different than reflection mode, see Fig. 3. This is because the attenuation is along the beam axis and there is no self-attenuation from the fuel rod because the detectors are not in direct line-of-sight of the fuel sample. The symmetry is caused by the cylindrical symmetry of the fuel rod. If there were spatial distribution other than our choice of a flat/homogeneous distribution, then the curve will be different and may not be symmetric (this also holds true for reflection). The curves plotted are of the various $\mathrm{Pu}$ concentrations (listed above) and are shown in different colors from $0.0 \%$, black to $1.0 \%$ in orange.

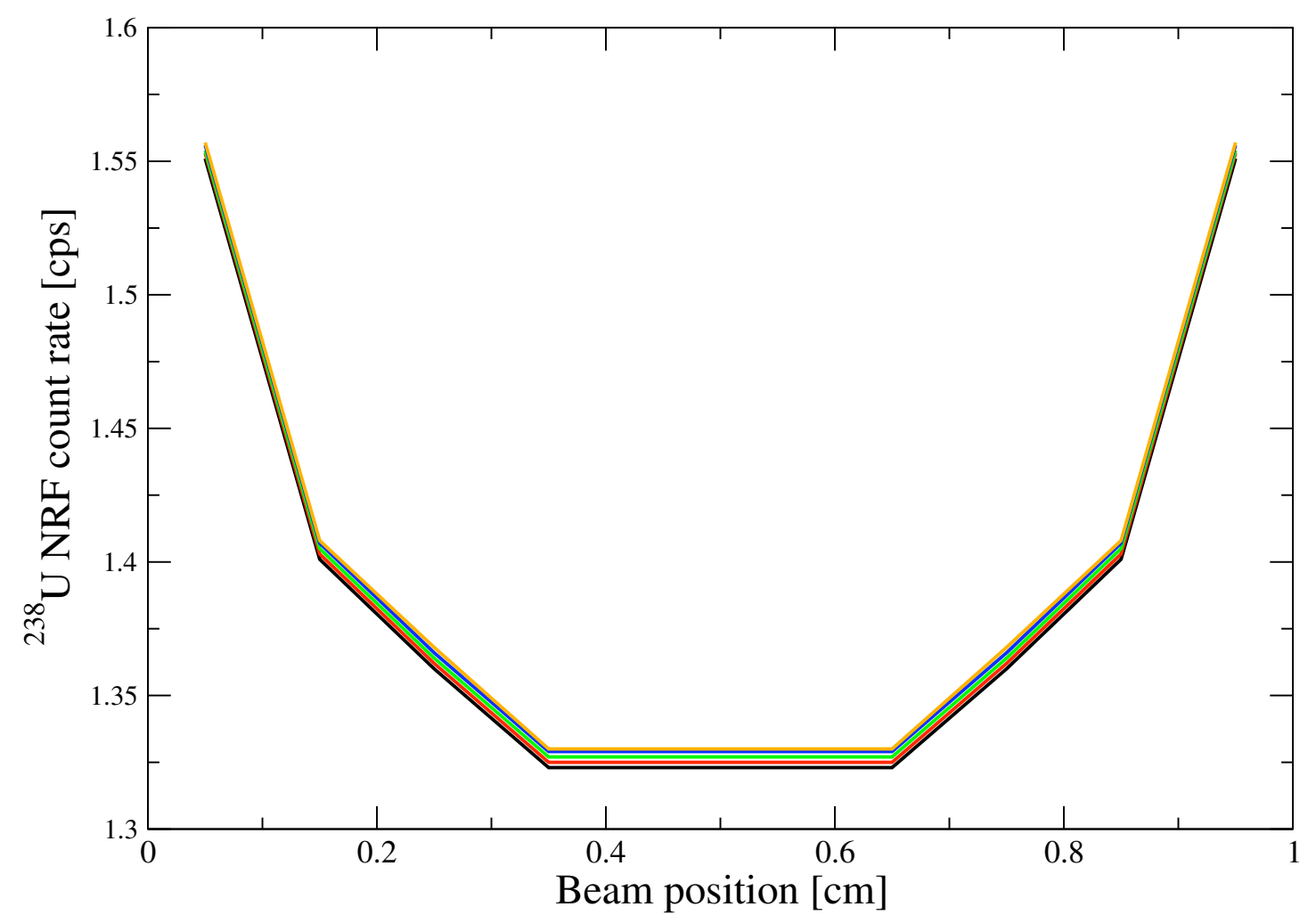

Figure 7: Plot of $238 \mathrm{U}$ NRF count rate of the transmission detector. The peak flux was assumed to be $400 / \mathrm{eV} / \mathrm{s}$. See text for more details.

Figure 8 is a plot of the $239 \mathrm{Pu}$ NRF count rate in transmission mode as a function of beam position. The trend of the counts as a function of beam position is explained in Fig 7. The functions plotted are of the various Pu concentrations (listed above) and are shown in different colors from $0.0 \%$, black to $1.0 \%$ in orange. The count rate in transmission mode is much higher than in reflection mode as seen in Fig. 4. 


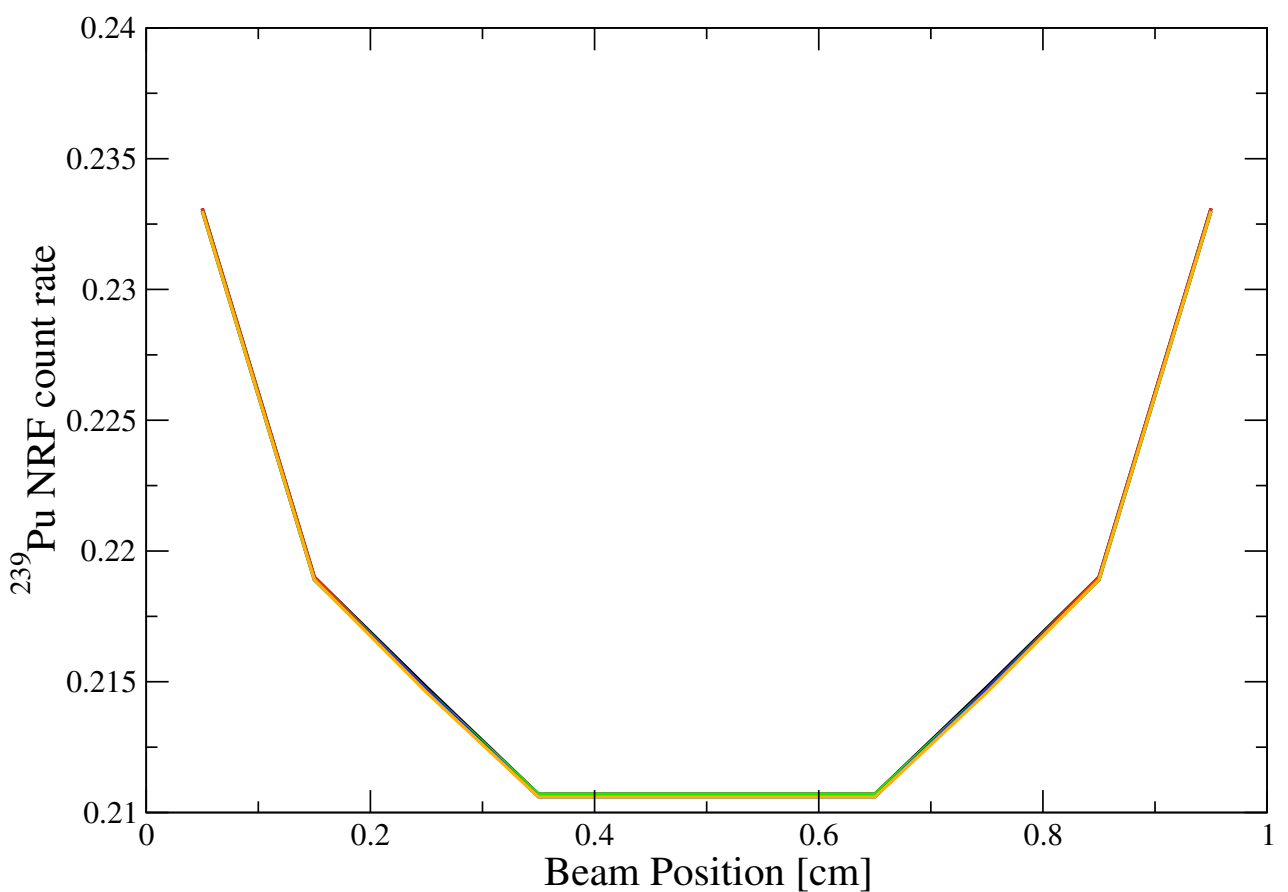

Figure 8: Plot of 239Pu NRF count rate with the transmission detector. The different curves represent different percentages of $\mathrm{Pu}$ (i.e. black $=0.0 \%$, red $=0.25 \%$, green $=0.5 \%$, blue $=0.75 \%$, orange $=1.0 \%$ ). The peak flux was assumed to be $400 / \mathrm{eV} / \mathrm{s}$. See text for more details.

Figure 9 is a plot of the $240 \mathrm{Pu}$ NRF count rate in transmission mode as a function of beam position. The trend of the counts as a function of beam position is explained in Fig 7. The functions plotted are of the various Pu concentrations (listed above) and are shown in different colors from $0.0 \%$, black to $1.0 \%$ in orange. 


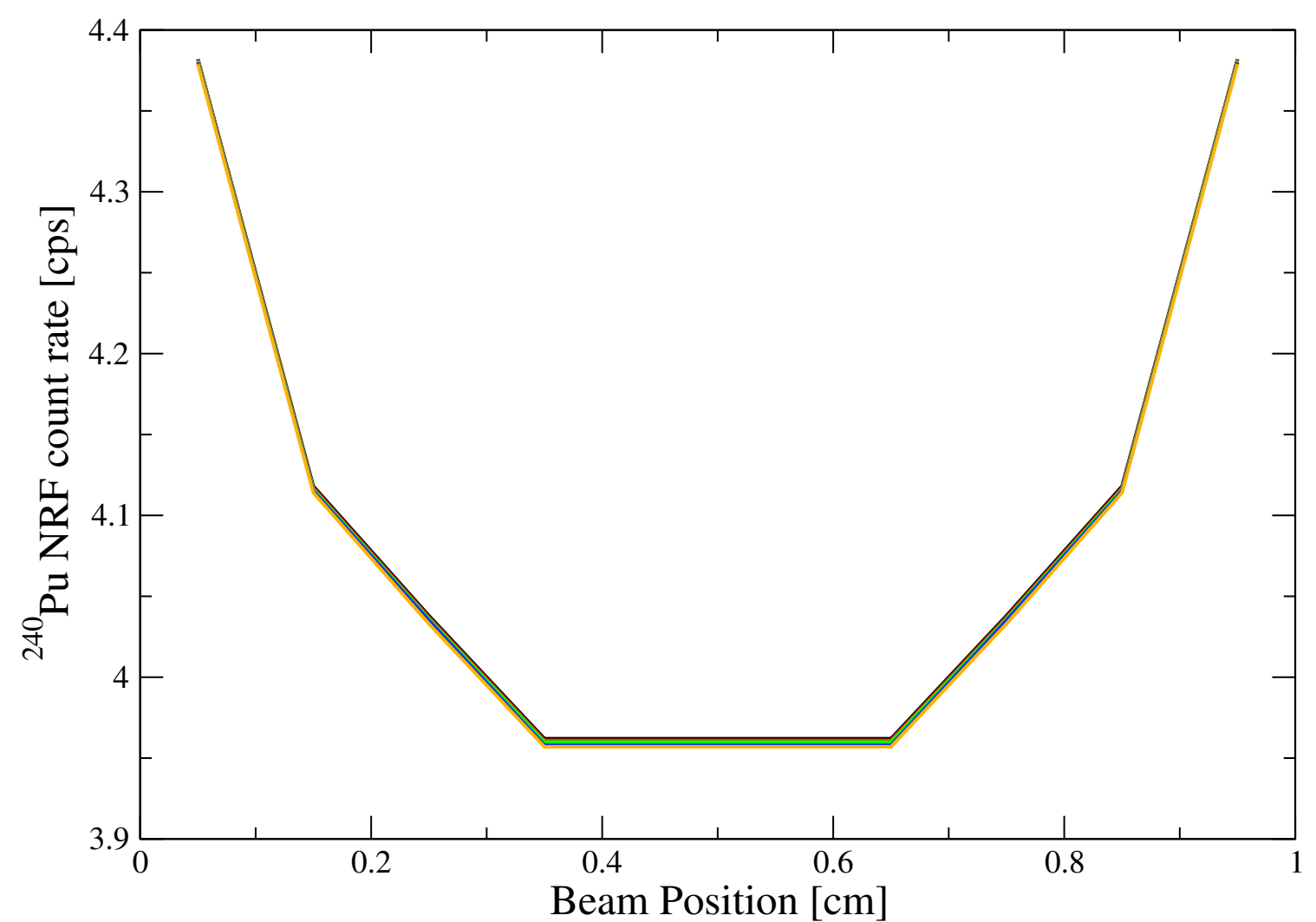

Figure 9: Plot of 240Pu NRF count rate with the transmission detector. The different curves represent different percentages of $\mathrm{Pu}$ (i.e. black $=0.0 \%$, red=0.25\%, green=0.5\%, blue $=0.75 \%$, orange=1.0\%). The peak flux was chosen to be $400 / \mathrm{eV} / \mathrm{s}$. See text for more details.

\section{Discussion:}

As alluded to above, there are certain metrics that may be useful to determining Pu content in spent nuclear fuel using NRF with quasi-monoenergetic sources. The overwhelming issue that has been spelled out is the uncertainties. The uncertainties can be quite large in some cases and may not have a remedy without some clever detection scheme (i.e. threshold detectors, gamma-ray imaging, etc...). In our introductory section on assay methods (See Sec. NRF-Based Assay Methods) we introduced two methods that would benefit here, the $r$-method and the $f$ method.

\section{r-method:}

To begin, we will look at the $r$-method. In figure 10, we observe the ratio of $239 \mathrm{Pu}$ to $238 \mathrm{U}$ NRF counts in reflection mode (i.e. Fig 4 divided by Fig 5). The curvature in Fig 4 is much greater than in Fig 5. The differences of curvature cause the ratio to have curvature of its own. If the curves in Figs 4 and 5 were the same, then the ratio of the two curves would give a straight line. The functions plotted are of the various Pu concentrations (listed above) and are shown in different colors from $0.0 \%$, black to $1.0 \%$ in orange. One can clearly see that for any given beam 
position, the ratio for each $\mathrm{Pu}$ concentration is equidistant from its neighboring value. That implies that curve that relates the ratio to $\mathrm{Pu}$ concentration is linear.

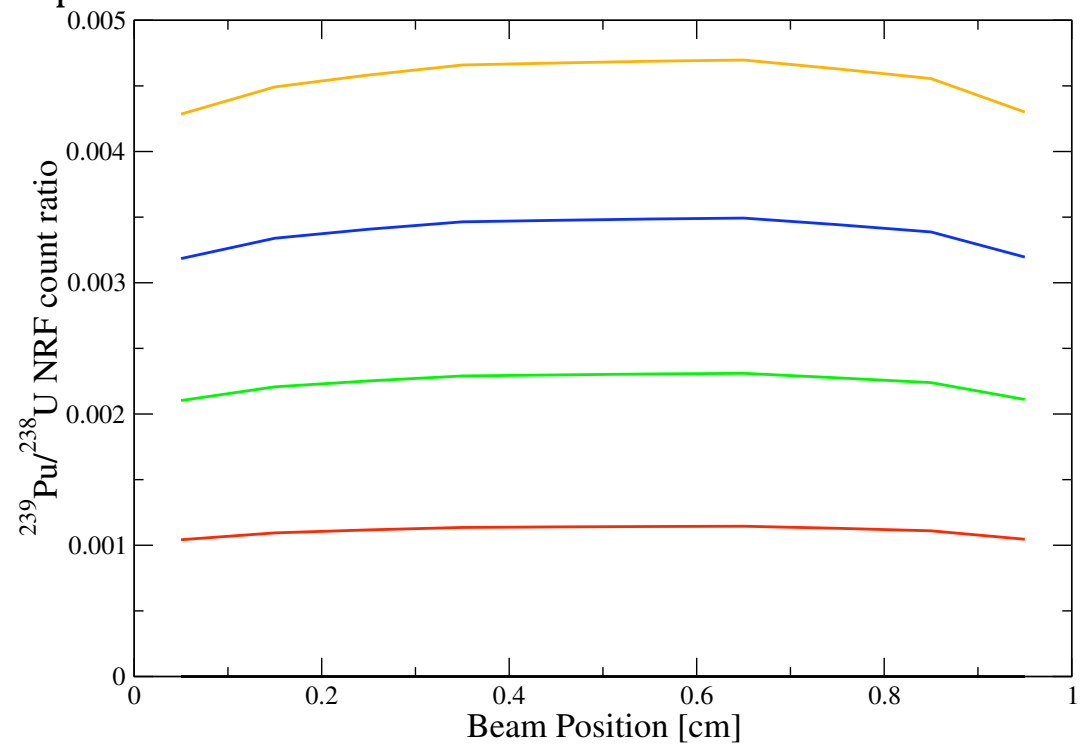

Figure 10: Plot of ratio of $239 \mathrm{Pu}$ to $238 \mathrm{U}$ in reflection mode. The beam position nearest to the detector suggests the best method to determining Pu content. The peak flux was chosen to be $400 / \mathrm{eV} / \mathrm{s}$. See text for more details.

Another possible method for determining $\mathrm{Pu}$ content is spent fuel is given in figure 11 , which is the ratio of $239 \mathrm{Pu}$ to $238 \mathrm{U}$ in transmission mode (i.e. Fig 8 divided by Fig 7). Similar to Fig 10, Fig 11 shows a linear dependence between the ratio (here, in transmission mode) and $\mathrm{Pu}$ concentration for a given beam position. This dependence allows us a method to determine the Pu concentration based solely on the ratio of counts.

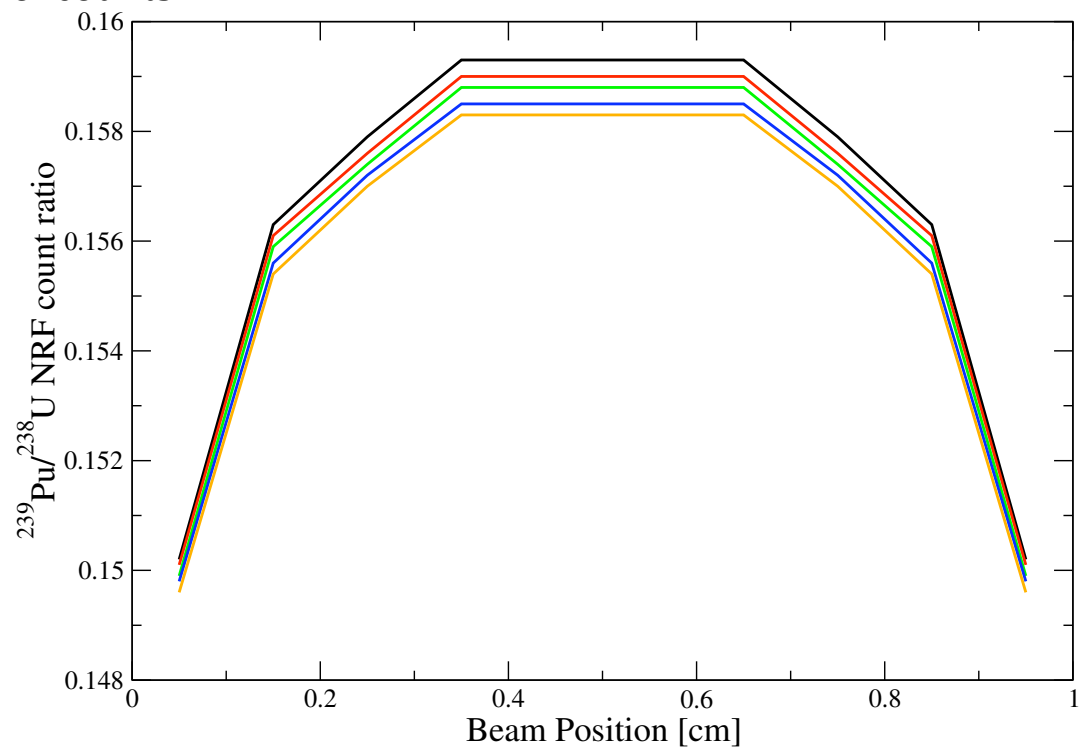

Figure 11: Plot of ratio of $239 \mathrm{Pu}$ to $238 \mathrm{U}$ in transmission mode. The beam position nearest to the center suggests the best method to determining Pu content because the spacing is the greatest. The peak flux was chosen to be $400 / \mathrm{eV} / \mathrm{s}$. See text for more details. 
The key question at this point is what are the counting times for NRF-based assay? When we insert the count rates and detected background rates (10 year old fuel radioactive backgrounds $\sim 0.02 / \mathrm{s} / \mathrm{eV} / \mathrm{g}$ at $2.5 \mathrm{MeV}$, calculated using ORIGEN, see [Her1986], and 20 year old Pu witness foil radioactive backgrounds $\sim 3 \times 10^{-}$ $6 / \mathrm{s} / \mathrm{eV} / \mathrm{g}$ at $2.5 \mathrm{MeV}$, calculated using GamGen, [Gos1990]) into Eq. 1, we find that $\mathrm{V}^{2}$ reflection $\sim 1 \times 10^{3}$ and $\mathrm{V}^{2}$ transmission $\sim 0.1$ for $239 \mathrm{Pu}$ for $1 . \%$ overall $\mathrm{Pu}$, see Tab. 6 . To determine the time to assay, Eq. 3, dictates that the slope is required. In reflection mode, the slope calculated from the values in Fig. 10 is about $10^{-2}$. In transmission mode, the slope from the values in Fig. 11 is about $10^{-3}$. The ad hoc count times are calculated and given in Tab. 6. Notice that the slope for $240 \mathrm{Pu}$ transmission is greater by a factor of 20 than $239 \mathrm{Pu}$, this implies a shorter assay time.

Table 6: Ad hoc values for estimated time to assay $1 . \%$ Pu to within $1 \%$ precision. Backgrounds were chosen to be $\sim 0.02 \mathrm{decays} / \mathrm{s} / \mathrm{eV} / \mathrm{g}$ for the fuel rod and $\sim 3 \times 10-6 \mathrm{decays} / \mathrm{s} / \mathrm{eV} / \mathrm{g}$ at $2.5 \mathrm{MeV}$ (these values are corrected for detector efficiency). These estimates are based on calculations for 10-year-old spent fuel (PWR) using ORIGEN [Her1986] and 20-year-old Pu (for the witness foils) using GamGen [Gos1990].

\begin{tabular}{|l|l|l|l|l|}
\hline Isotope & $\begin{array}{l}\text { Reflection } \\
\text { Rate [cps] }\end{array}$ & $\mathrm{V}^{2}$ reflection & $\begin{array}{l}\text { Slope for } \\
\text { reflection }\end{array}$ & $\begin{array}{l}\text { Reflection Assay time } \\
{[\mathrm{sec}]}\end{array}$ \\
\hline $238 \mathrm{U}$ & $\sim 0.11$ & & & \\
\hline $239 \mathrm{Pu}$ & $\sim 0.0005$ & $\sim 1 . \mathrm{x} 10^{3}$ & $\sim 0.005$ & $\sim 5 \times 10^{15}$ \\
\hline $240 \mathrm{Pu}$ & $\sim 0.0007$ & $\sim 1 . \mathrm{x}^{3}$ & $\sim 0.007$ & $\sim 2 \times 10^{15}$ \\
\hline Isotope & $\begin{array}{l}\text { Transmission } \\
\text { Rate [cps] }\end{array}$ & $\mathrm{V}^{2}$ transmission & $\begin{array}{l}\text { Slope for } \\
\text { transmission }\end{array}$ & $\begin{array}{l}\text { Transmission Assay time } \\
{[\mathrm{sec}]}\end{array}$ \\
\hline $238 \mathrm{U}$ & $\sim 1.325$ & & & $\sim 1 \times 10^{13}$ \\
\hline $239 \mathrm{Pu}$ & $\sim 0.21$ & $\sim 0.1$ & $\sim 0.001$ & $\sim 1 \times 10^{12}$ \\
\hline $240 \mathrm{Pu}$ & $\sim 3.95$ & $\sim 6$ & $\sim 0.02$ & \\
\hline
\end{tabular}

We have plotted our count times for the $r$-method assay times in Fig. 12. The black and red curves in Fig. 12 are the counting times in reflection mode one expects at a HIGS-like facility for the $239 \mathrm{Pu} / 238 \mathrm{U}$ ratio and $240 \mathrm{Pu} / 238 \mathrm{U}$ ratio, respectively. The blue and green curves in Fig. 12 are the counting times in transmission mode one expects at a HIGS-like facility for the $239 \mathrm{Pu} / 238 \mathrm{U}$ ratio and $240 \mathrm{Pu} / 238 \mathrm{U}$ ratio, respectively.

We may be skeptical of the reflection assay times because they are based on realistic calculations and not on empirical data. More refined data may shift the reflection mode curves to lower assay times. A priori one might expect the reflection mode to be more advantageous over transmission since these application doses not limit the dose of the source in contrast to detection systems using NRF. (This is because of the ever-present number of workers in a scanning area where NRF-based technologies may be deployed.) 


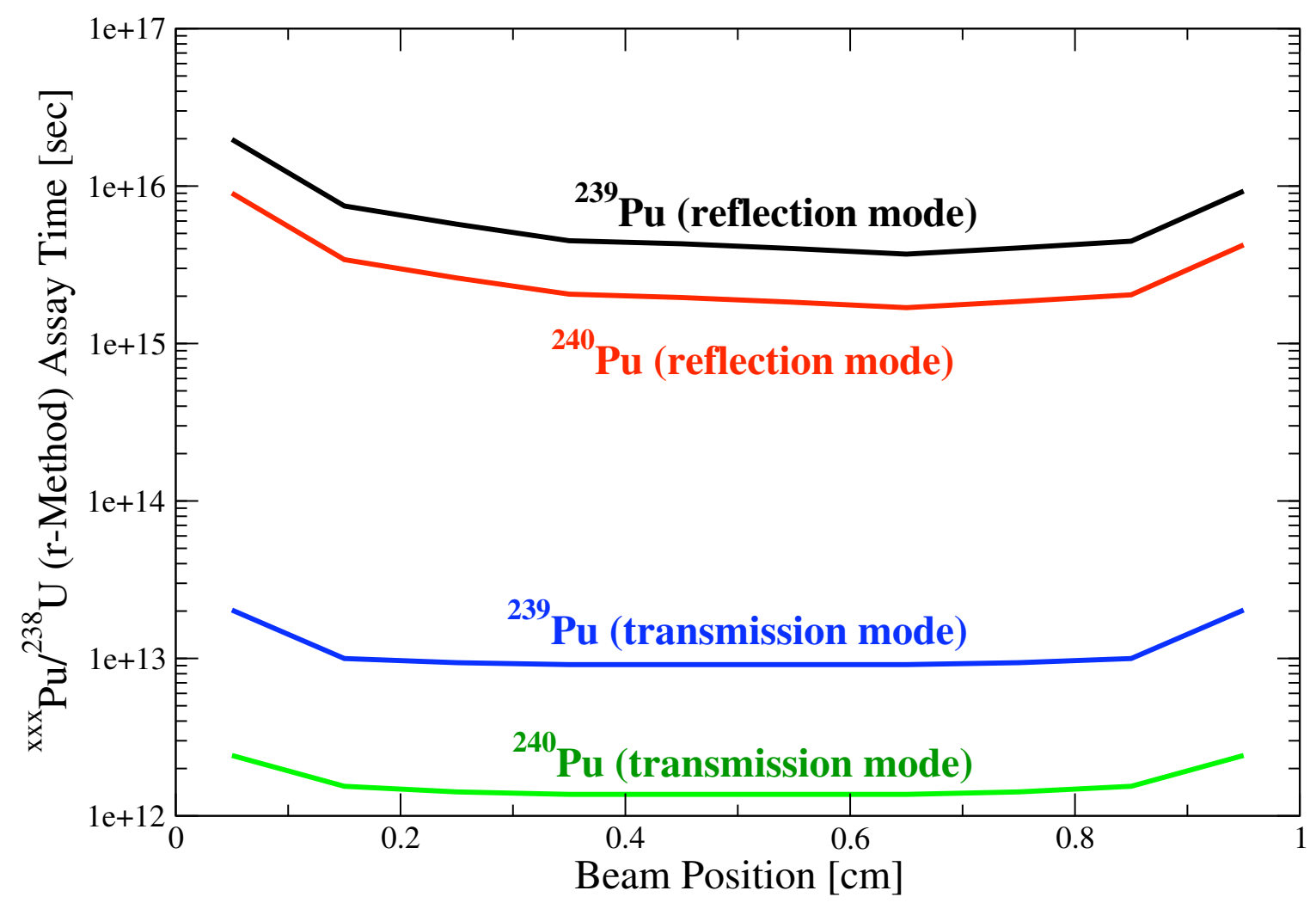

Figure 12: Plot of count times for various $P u$ to $238 \mathrm{U}$ ratios. Black and green curves are count times for $239 \mathrm{Pu} / 238 \mathrm{U}$ and $240 \mathrm{Pu} / 238 \mathrm{U}$ in reflection mode, respectively. Red and blue curves are count times for $239 \mathrm{Pu} / 238 \mathrm{U}$ and $240 \mathrm{Pu} / 238 \mathrm{U}$ in transmission mode, respectively. The peak flux was assumed to be $400 / \mathrm{eV} / \mathrm{s}$. Pu concentration is $1 \%$. Precision is $1 \%$.

We now want to go into detail with the $r$-method and see where if at all we can optimize things and/or where this type of NRF-based assay performs the best.

\section{Reflection}

To begin we look at the reflection mode for the $r$-method. We note from Eq. 2 that the dominant term is the first term and we can write:

$$
V^{2} \approx \frac{R_{P u}+B_{P u}}{S_{U}^{2}}=\frac{S_{P u}+2 B_{P u}}{S_{U}^{2}} \approx \frac{S_{P u}+2 B G \Gamma}{S_{\text {Equation 10 }}^{2}}=r^{2} \frac{S_{P u}+2 B G \Gamma}{S_{P u}^{2}}
$$

where $B G$ is defined as the radioactive background of the spent fuel (assumed to be the greatest contributor to the overall background) in units of decays $/ \mathrm{sec} / \mathrm{g}$, $\Gamma$ is defined to be the number of eV per detector bin, which we assume to be $\sim 1 \mathrm{keV}$ wide (implying that $\Gamma \sim 1000 \mathrm{eV}$ ), $S$ is defined as the NRF signal rate (see Eq. 2 ), and $r$ is the ratio of the Pu NRF signal rate to the U NRF signal rate.

At this point there are only two possibilities. First, the source strength is not strong enough to overcome the background rate. This implies that $\mathrm{S}_{\mathrm{Pu}}$ in Eq. 10 is negligible to BG. In that case, the assay times would be infinite and we can write it (from Eq. 4): 


$$
t \approx \frac{1}{p^{2}} \cdot \frac{2 B G \Gamma}{(\rho L)_{f u e l}^{2} n^{2}(\varepsilon \alpha)^{2}\left(\frac{N_{A}}{M_{P u}}\right)^{2} \Phi_{0}^{2}}=\frac{1}{n^{2} p^{2}} \cdot \frac{2 B G \Gamma^{3} m_{f u e l} M_{P u}^{2}}{(\rho L)_{f u e l}^{2}(\varepsilon \alpha \Gamma)^{2}\left(N_{A}\right)^{2} \Phi_{0}^{2} \sigma_{N R F}^{2}}
$$

where $M_{P u}$ is the molar mass of Pu, $m_{f u e l}$ is the total mass of the assay sample in grams, and $(\rho L)_{\text {Fuel }}$ is the fuel thickness. Notice that we have chosen to write the quantity, $(\varepsilon \alpha \Gamma)$. The (absorption) quantity, $\alpha$, contains the self-absorption of the sample as well as the absorption of any detector filter. We will define the latter to be $\alpha_{0}$. For HPGe detectors the quantity $\left(\varepsilon \alpha_{0} \Gamma\right)$ is approximately 1 . Notice in Eq. 11, we made use of the relationship $r=m n+b$, where for reflection mode, $b \sim 0$, which implies $r \sim m n$.

What is interesting in Eq. 11 is that the assay time improves as $1 / \Phi^{2} . A$ priori, we would have expected that the time would have improved as $1 / \Phi$, but since the backgrounds have been assumed to be dominate we see that this is not the case. It is a moot point though because the signal rate may be too low to even be observed.

The second possibility is that the flux is strong enough such that the NRF signal and flux induced backgrounds dominate the radioactive background of the fuel sample. In this case the quantity $V^{2}$ is:

$$
V^{2} \approx r^{2} \frac{S_{P u}+2 R_{\text {elastic }}}{S_{P u}^{2}}=\frac{r^{2}}{S_{P u}^{2}} S_{P u}\left(1+2 \frac{R_{\text {elastic }}}{S_{P u}}\right) \rightarrow \frac{r^{2}}{S_{P u}}\left(1+2 \frac{\Gamma \sigma_{\text {elastic }}}{n \sigma_{N R F}}\right)
$$

where we have ignored the now negligible $B G$ and $\sigma_{\text {elastic }}$ is the elastic cross-section from the fuel (e.g. Delbruck, Rayleigh, Thomson).

From Eq. 12 the new assay time would be:

$$
t=\frac{V^{2}}{(m n p)^{2}} \approx \frac{1}{p^{2}} \cdot \frac{2 \Gamma M_{\text {Fuel }}}{n(\rho L)_{\begin{array}{c}
\text { Fuel } \\
\text { Equation } 13
\end{array}}(\varepsilon \alpha \Gamma) N_{A} \Phi_{0} \sigma_{N R F}}\left(1+2 \frac{\Gamma \sigma_{\text {elastic }}}{n \sigma_{N R F}}\right)
$$

Although the radioactive background has been mitigated, we are left with an expression that is difficult to carry-on any further. The reason is that the attenuation factor, $\alpha$, is a function of self-absorption of the fuel. It is unclear the path the photons will take within the fuel to get to the detector and subsequently the absorption factor becomes a large uncertainty. For a single fuel rod there are ways around this issue (e.g. transport codes, Monte-Carlo or something similar to the above simulations) but the goal of our discussion is to study full assemblies. The complications that arise from the non-homogeneity of the fuel assembly provide a challenge we will leave for follow-on research. 


\section{Transmission}

We wish now to discuss the $r$-method using the transmission detection mode and ways to optimize the system to lessen the assay time. The derivation of the $r$ method transmission-mode assay time (similar to Eq. 13) is not a simple task and we will leave out many steps that enthusiastic readers may wish to fill in on their own. We start the derivation by noting the slope for the ratio method:

$$
\begin{aligned}
& m=\frac{\Delta r}{\Delta n}=\frac{\frac{S_{P u}(n)}{S_{U}(n)}-\frac{S_{P u}(0)}{S_{U}(0)}}{n-0} \\
& \Rightarrow m=\frac{1}{n} \cdot\left(\frac{\Phi_{P u}^{\prime}(n)(\varepsilon \alpha)_{P u}(\rho l)_{P u} \frac{N_{A}}{M_{P u}} \sigma_{P u}}{\Phi_{U}^{\prime}(n)(\varepsilon \alpha)_{U}(\rho l)_{U} \frac{N_{A}}{M_{U}} \sigma_{U}}-\frac{\Phi_{P u}^{\prime}(0)(\varepsilon \alpha)_{P u}(\rho l)_{P u} \frac{N_{A}}{M_{P u}} \sigma_{P u}}{\Phi_{U}^{\prime}(0)(\varepsilon \alpha)_{U}(\rho l)_{U} \frac{N_{A}}{M_{U}} \sigma_{U}}\right) \\
& \Rightarrow m=\frac{1}{n} \cdot \frac{\Phi_{P u}^{\prime}(n)(\varepsilon \alpha)_{P u}(\rho l)_{P u} \frac{N_{A}}{M_{P u}} \sigma_{P u}}{\Phi_{U}^{\prime}(n)(\varepsilon \alpha)_{U}(\rho l)_{U} \frac{N_{A}}{M_{U}} \sigma_{U}} \cdot\left(1-\frac{\Phi_{P u}^{\prime}(0) \Phi_{U}^{\prime}(n)}{\Phi_{P u}^{\prime}(n) \Phi_{U}^{\prime}(0)}\right) \\
& \rightarrow m \approx \frac{1}{n} \cdot \frac{\Phi_{P u}^{\prime}(n)(\varepsilon \alpha)_{P u}(\rho l)_{P u} \frac{N_{A}}{M_{P u}} \sigma_{P u}}{\Phi_{U}^{\prime}(n)(\varepsilon \alpha)_{U}(\rho l)_{U} \frac{N_{A}}{M_{U}} \sigma_{U}} \cdot\left(n \sigma_{P u} \frac{N_{A}}{M_{F u e l}}(\rho L)_{F u e l}\right) \\
& \text { Equation 14}
\end{aligned}
$$

where $(\varepsilon \alpha)_{\mathrm{x}}$ and $(\rho l)_{\mathrm{x}}$ are the detector (efficiency and filter) and witness foil (thickness) characteristics for isotope $x . \sigma_{\mathrm{x}}$ is the NRF cross-section of isotope $x$. The last step in Eq. 14 was accomplished by noting for our study, $n<<1$, which implies that the flux after the fuel sample for $\mathrm{U}$ is approximately the same for $n=0$ and $n \neq 0$. Furthermore, we note that:

$$
\Phi_{P u}^{\prime}(n)=\Phi_{P u}^{\prime}(0) e^{-\mu_{P u-N R F}(n) \cdot(\rho L)_{F u e l}}
$$

Since we claim that $n<<1$ we can expand the exponential in Eq. 15, substitute into the third step in Eq. 13, keeping only the leading term (since all higher order terms will be negligible), and expand the nuclear attenuation coefficient to get the final step in Eq. 14. Note that in a real-world deployment, an operator would not use Eq. 14, but rather determine $m$ by calibration methods using different $(\rho L)$ calibration-foils and different $n$. The idea is to measure $m$ to a very high precision to reduce the systematic uncertainties, which can easily be done off-line.

Our next step to deriving the $r$-method transmission-mode assay time (Eq. 4) is to determine $V^{2}$ from Eq. 2. For this step we will include the LO term and the NLO term in Eq. 2, essentially the whole of Eq. 2. As in our discussion of the reflection 
mode, we will assume the beam strength after the fuel sample is large enough so that the natural background radiation is negligible. This is easily achievable since the calculations for the background radiation are quite small, $\sim 3 \times 10^{-6} / \mathrm{s} / \mathrm{eV} / \mathrm{g}$ at 2.5 $\mathrm{MeV}$, calculated using GamGen, [Gos1990]. We can compare the backgrounds to the NRF signal rate with a HIGS-type source, see Fig 8 . We begin by deriving the LO term for $V^{2}$ :

$$
\begin{aligned}
& V_{L O}^{2}=\frac{S_{P u}^{2}}{S_{U}^{4}} \cdot\left(R_{U}+B_{U}\right)=\frac{S_{P u}^{2}}{S_{U}^{4}} \cdot\left(S_{U}+2 B G+2 R_{\text {elastic }}\right) \stackrel{\Phi \sim L A R G E}{\longrightarrow} \frac{S_{P u}^{2}}{S_{U}^{4}} \cdot\left(S_{U}+2 R_{\text {elastic }}\right) \\
& \Rightarrow V_{L O}^{2} \approx \frac{\left(\Phi_{P u}^{\prime}(n)(\varepsilon \alpha)_{P u}(\rho l)_{P u} \frac{N_{A}}{M_{P u}} \sigma_{P u}\right)^{2}}{\left(\Phi_{U}^{\prime}(n)(\varepsilon \alpha)_{U}(\rho l)_{U} \frac{N_{A}}{M_{U}} \sigma_{U}\right)^{4}} \cdot \Phi_{U}^{\prime}(n)(\varepsilon \alpha)_{U}(\rho l)_{U} \frac{N_{A}}{M_{U}} \Gamma \sigma_{\text {elastic }}\left(2+\frac{\sigma_{U}}{\Gamma \sigma_{\text {elastic }}}\right)
\end{aligned}
$$

Equation 16

where we have assumed that the flux impinging on the witness foil is large so that the radioactive background at the resonance region is negligible.

We can now use Eqs. 15 and 16 to derive the $r$-method transmission-mode assay time to leading order:

$$
\begin{aligned}
& t_{L O} \approx \frac{1}{n^{2} p^{2}} \cdot \frac{1}{\Phi_{U}^{\prime}(n)} \cdot \frac{1}{(\rho l)_{U}} \cdot \frac{M_{U} M_{\text {Fuel }}^{2}}{N_{A}^{3}} \cdot \frac{1}{\Gamma(\varepsilon \alpha)_{U}} \cdot \frac{\Gamma^{2} \sigma_{\text {elastic }}}{\sigma_{P u}^{2} \sigma_{U}^{2}} \cdot \frac{1}{(\rho L)_{\text {Fuel }}^{2}} \cdot\left(2+\frac{\sigma_{U}}{\Gamma \sigma_{\text {elastic }}}\right) \\
& \rightarrow t_{L O}=\frac{1}{n^{2} p^{2}} \cdot \frac{1}{\Phi_{U}} \cdot \frac{M_{U} M_{\text {Fuel }}^{2}}{N_{A}^{3}} \cdot \frac{1}{\Gamma\left(\varepsilon \alpha_{0}\right)_{U}} \cdot \frac{\Gamma^{2} \sigma_{\text {elastic }}}{\sigma_{P u}^{2} \sigma_{U}^{2}} \cdot\left(2+\frac{\sigma_{U}}{\Gamma \sigma_{\text {elastic }}}\right) \cdot f\left((\rho l)_{U},(\rho L)_{\text {Fuel }} ; U\right)
\end{aligned}
$$

Equation 17

Where $f\left((\rho l)_{U},(\rho L)_{\text {Fuel; }} U\right)$ is a function of areal thickness of the uranium witness foil and the fuel and is found simply substituting in the following expressions:

$$
\begin{aligned}
& \Phi_{U}^{\prime}(n)=\Phi_{U} e^{-\left(\mu_{U-N R F}(n)+\mu_{A-F \text { - uel }}\right)(\rho L)_{\text {Fuel }}} \text { and } \\
& (\alpha)_{U}=\alpha_{0} e^{-\left(\frac{3}{2} \mu_{U-N R F}+\frac{5}{2} \mu_{U-W F}\right)(\rho l)_{U}}
\end{aligned}
$$

Equation 18

$\mu_{\mathrm{U}-\mathrm{NRF}}(\mathrm{n})$ and $\mu_{\mathrm{A} \text {-Fuel }}$ is the nuclear and atomic attenuation through the fuel, respectively, and $\mu_{\mathrm{U}-\mathrm{NRF}}$ and $\mu_{\mathrm{U}-\mathrm{WF}}$ is the nuclear and atomic attenuation through the witness foil, respectively. Equation 18 assumes an average detector angle at 120degrees in the lab frame and that the average attenuation for the witness foil is for half the thickness of the witness foil. Recall that $\alpha_{0}$ is the attenuation of any absorbers/filters placed in front of the detectors to reduce low energy noise and radioactive backgrounds and not the attenuation from the witness foil, i.e. Eq. 18 .

We write the NLO term for the $r$-method transmission-mode assay time and leave the complete derivation for the reader: 


$$
t_{N L O}=\frac{1}{n^{2} p^{2}} \cdot \frac{1}{\Phi_{P u}} \cdot \frac{M_{P u} M_{F u e l}^{2}}{N_{A}^{3}} \cdot \frac{1}{\Gamma\left(\varepsilon \alpha_{0}\right)_{P u}} \cdot \frac{\Gamma^{2} \sigma_{\text {elastic }}}{\sigma_{P u}^{4}} \cdot\left(2+\frac{\sigma_{P u}}{\Gamma \sigma_{\text {elastic }}}\right) \cdot f\left((\rho l)_{P u},(\rho L)_{F u e l} ; P u\right)
$$

Equation 19

If we sum Eqs. 17 and 19, we get the total $r$-method transmission-mode assay time:

$$
t_{r}=\frac{1}{n^{2} p^{2}} \cdot \frac{1}{\Phi_{U}} \cdot \frac{M_{U} M_{\text {Fuel }}^{2}}{N_{A}^{3}} \cdot \frac{1}{\Gamma\left(\varepsilon \alpha_{0}\right)_{U}} \cdot \frac{\Gamma^{2} \sigma_{\text {elastic }}}{\sigma_{U}^{2} \sigma_{P u}^{2}} \cdot\left(2+\frac{\sigma_{U}}{\Gamma \sigma_{\text {elastic }}}\right) \cdot h\left((\rho l)_{U},(\rho l)_{P u},(\rho L)_{\text {Fuel }}\right)
$$

Equation 20

Where $h\left((\rho l)_{U},(\rho l)_{P u},(\rho L)_{F u l l}\right)$ is a function of areal thickness of the uranium and plutonium witness foils and the fuel.

We note that for both functions above (Eqns. 17 ad 19) that there is a dependence on the spectral shape of the beam. Notice that if there is negligible flux on the Pu channel (i.e. $\Phi_{\mathrm{Pu}} \sim 0$ ) then the counting time goes to infinity, which we expect because there are no signals being created in that case. This can happen if the beam is centered on the $U$ resonance and is too narrow to extend any appreciable flux to the Pu resonance. The corollary is also true (except in the denominator of $t_{\mathrm{NLO}}$ ), which is to say if there is no appreciable flux on the $\mathrm{U}$ resonance (i.e. $\Phi_{U} \sim 0$ ), the counting time goes to infinity.

We note that if we tune our beam to the Pu resonance, i.e. $\Phi_{\mathrm{Pu}}=\Phi_{0}$, then the flux on the $\mathrm{U}$ resonance is:

$$
\begin{aligned}
& \text { Quasi-Monoenergetic Source }: \Phi_{U}=\Phi_{0} e^{-\left(\frac{\left(E_{U}-E_{P_{u}}\right)^{2}}{2 \Sigma^{2}}\right)} \\
& \text { or } \\
& \text { Bremsstrahlung Source: } \Phi_{U}=\Phi_{0} \cdot\left(\frac{E_{e}-E_{U}}{E_{e}-E_{P u}}\right)
\end{aligned}
$$

Equation 21

where $\Sigma$ is the width of the quasi-monoenergetic beam (assumed to be Gaussian) and $E_{\mathrm{e}}$ is the end-point energy of the bremsstrahlung source. We have used the spectral form for bremsstrahlung found in [Dys1959] and references therein. We will come back to spectral shape when we compare the $r$-method and the $f$-method, but for now we will continue with our optimization procedure.

To choose the appropriate thickness of witness foil and prevent selfattenuation of the NRF signal being sought we need to find the minimum of Eq. 20 with respect to $(\rho l)_{\mathrm{U}}$ and $(\rho l)_{\mathrm{Pu}}$. We differentiate Eq. 20 with respect to $(\rho l)_{\mathrm{U}}$ and $(\rho l)_{\mathrm{Pu}}$ and we find that the assay time is minimized when $(\rho l)_{\mathrm{U}}=\left(3 / 2 \mu_{\mathrm{U}-\mathrm{NRF}}+5 / 2 \mu_{\mathrm{U}}\right.$ - 
$\mathrm{WF}^{-1}$ and $(\rho l)_{\mathrm{Pu}}=\left(3 / 2 \mu_{\mathrm{Pu}-\mathrm{NRF}}+5 / 2 \mu_{\mathrm{Pu}-\mathrm{WF}}\right)^{-1}$. A quick calculation shows that $(\rho l)_{\mathrm{U}} \sim$ $5.68 \mathrm{~g} / \mathrm{cm}^{2}$ and $(\rho l)_{\mathrm{Pu}} \sim 7.14 \mathrm{~g} / \mathrm{cm}^{2}$, or $l_{\mathrm{U}} \sim 2.8 \mathrm{~mm}$ and $l_{\mathrm{Pu}} \sim 3.6 \mathrm{~mm}$. These thicknesses are approximately equivalent to the thickness used for the simulations in the above figures.

Figure 13 shows the plot of Eq. 20 (assay time per source photon) with the optimized witness foil thicknesses. Figure 13 includes the time to assay $239 \mathrm{Pu}$ (black) and 240Pu (red) as a function of fuel thickness. Denoted in Fig. 13 are the fuel thicknesses of a single rod and the approximate thickness of a full 17x17 PWR assembly.

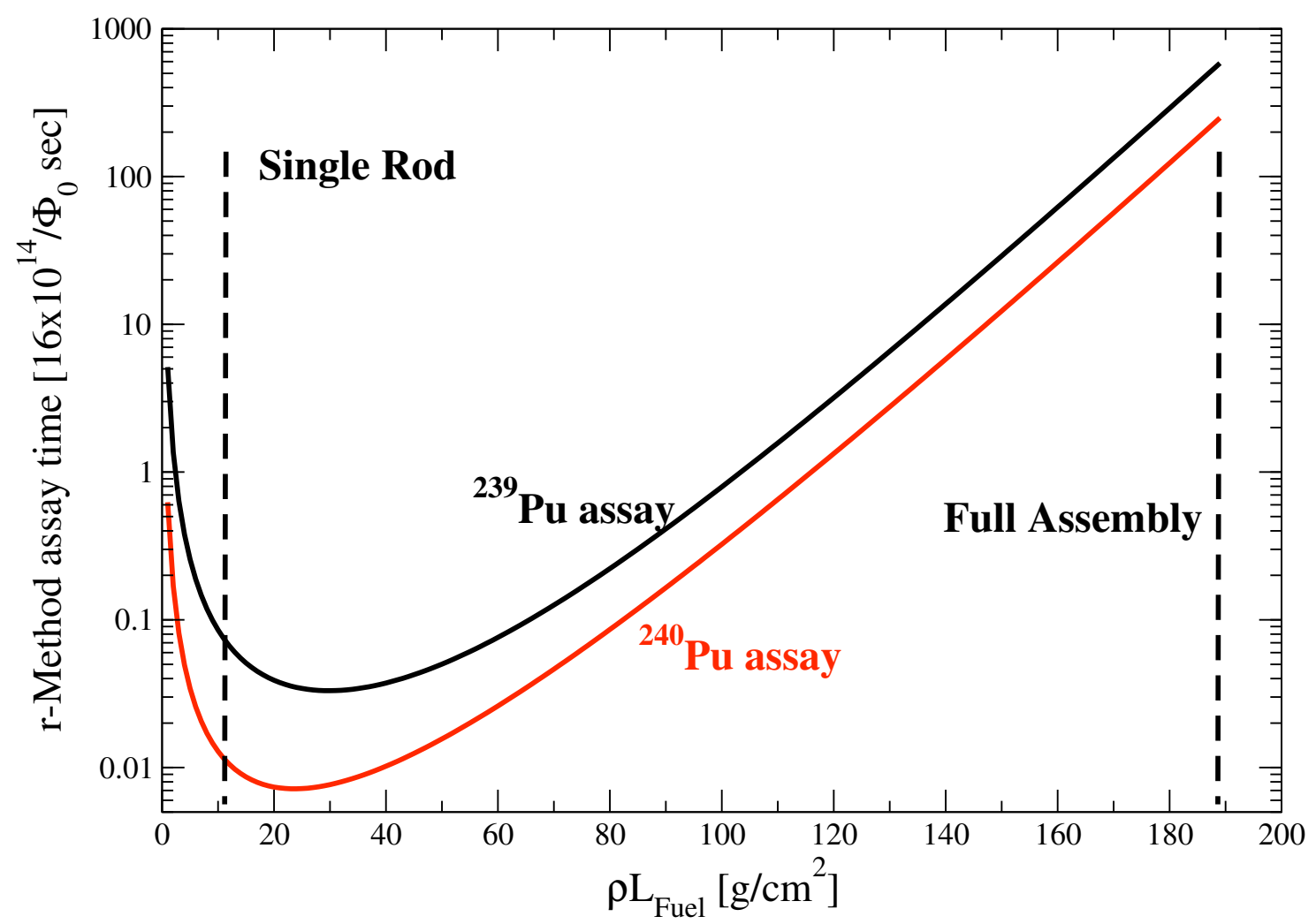

Figure 13: Plot of r-Method assay time for the transmission method per source photon as a function of fuel thickness. The units of the source photon are $1 / \mathrm{eV} / \mathrm{sec}$. Depicted above are the thicknesses of a single rod and a full assembly (17x17 PWR). Pu concentration is 1\%. Precision is $1 \%$.

The assay time for $239 \mathrm{Pu}$ is slightly higher compared to the time to assay $240 \mathrm{Pu}$ for all fuel thicknesses. We can see evidence of a shorter assay time for $240 \mathrm{Pu}$ in the location of the minimum, which is slightly shorter meaning the optical length through the fuel for $240 \mathrm{Pu}$ is shorter than $239 \mathrm{Pu}$. If the $240 \mathrm{Pu}$ content were a factor of 4 greater than what our initial assumption was (6\%), then the count times for 240Pu would decrease by a factor of 16.

The differential between the single rod and fuel assembly is mostly caused by the atomic attenuation of the fuel. According to Fig. 13 assay an entire PWR fuel assembly in one day would require a flux to be about $10^{17}$ photons/eV/sec on resonance. Notice also in Fig. 13 that the minimum assay time for the $r$-method is 
around $23 \mathrm{~g} / \mathrm{cm}^{2}$. If we changed the scenario to assay $235 \mathrm{U}$ instead of Pu at an IE of $\sim 3 \%$ and to a precision value of $3 \%$ and used the strongest NRF state at $1.733 \mathrm{MeV}$ [Ber2008] and use a source that is $\sim 10^{6}$ photons/eV/sec (in development at LLNL [Bar2009, Bar2011]), we could perform assay on a single pin in about $10^{3}$ seconds (i.e. 20 minutes).

f-method:

Now we turn our attention to the $f$-method. Figure 14 shows the calculated count times to determine isotopic mass of 239Pu (black) and 240Pu (red) to within $1 \%$ precision using our HIGS assumptions of a peak photon flux of $400 / \mathrm{eV} / \mathrm{sec}$ at 2.5 $\mathrm{MeV}$. Notice that 240Pu has a faster assay time than 239Pu by about a factor of 2 .

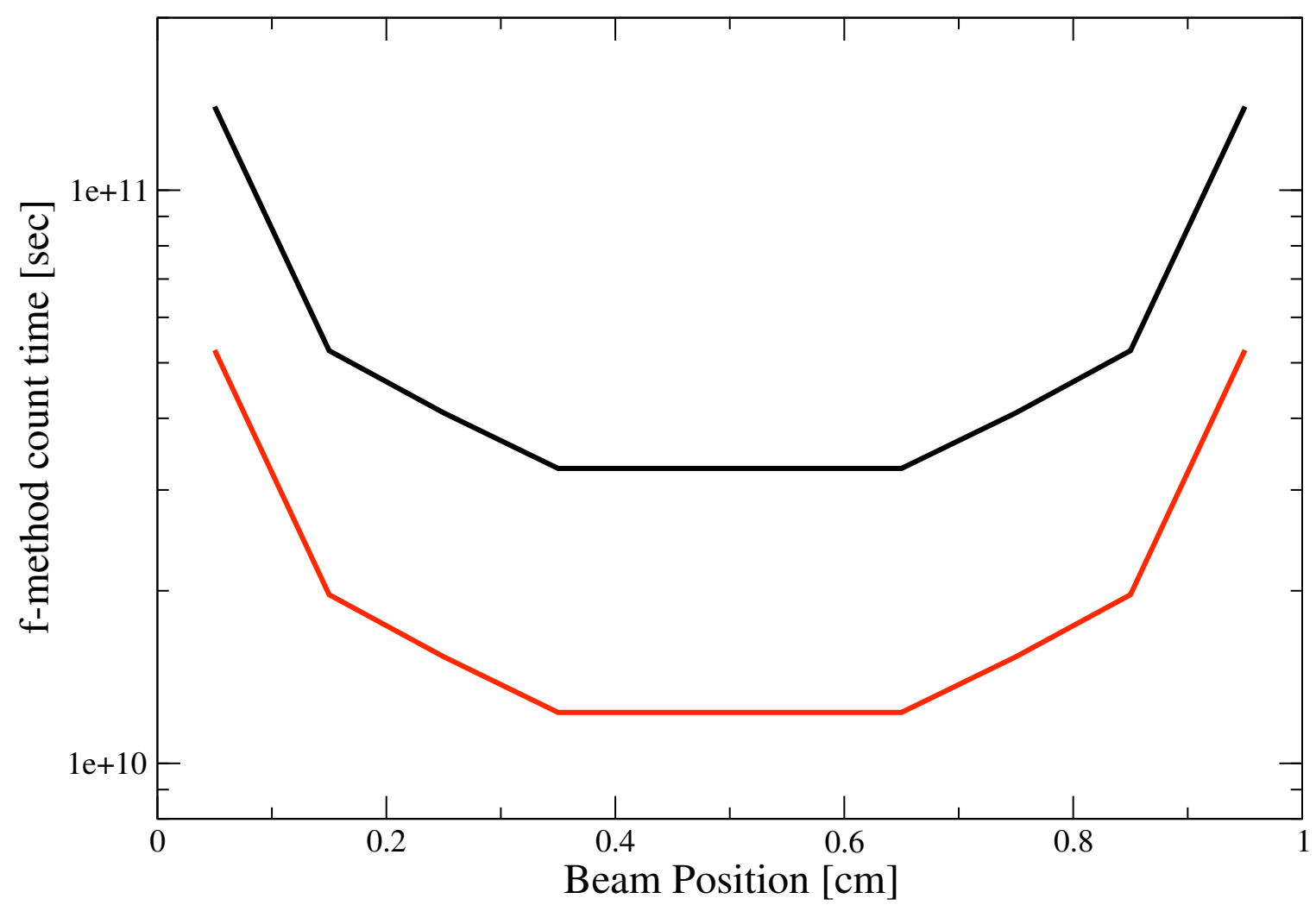

Figure 14: Plot of count time to determine mass of 239Pu (black), and 240Pu (red) in a spent fuel rod using the f-method. The peak flux was assumed to be $400 / \mathrm{eV} / \mathrm{s}$. The Pu concentration was $0.5 \%$. Precision is $1 \%$.

We proceed as before to optimize the $f$-method assay time in Fig. 14. We begin by solving Eq. 7 for $\ln \left(\mathrm{M} / M_{0}\right)$ and plugging into Eq. 9 . We then get the $\mathrm{LO}$ and NLO of $v^{2}$ in Eq. 8 and plug into Eq. 8 to get: 


$$
\begin{aligned}
& t_{f} \approx \frac{1}{n^{2} p^{2}} \cdot\left(\frac{M_{\text {Fuel }}}{N_{A}}\right)^{2} \cdot \frac{1}{\sigma_{P_{u}}^{2}} \cdot \frac{1}{(\rho L)_{\text {Fuel }}^{2}} \cdot\left(\frac{S_{\text {elassic }}^{\prime}+2 B G}{\left(S_{\text {elastic }}^{\prime}\right)^{2}}+\frac{S_{P_{u}}^{\prime}+S_{\text {elastic }}^{\prime}+2 B G}{\left(S_{P_{u}}^{\prime}+S_{\text {elassic }}^{\prime}\right)^{2}}\right) \\
& \stackrel{\Phi \sim L A R G E}{\longrightarrow} \frac{1}{n^{2} p^{2}} \cdot\left(\frac{M_{\text {Fuel }}}{N_{A}}\right)^{2} \cdot \frac{1}{\sigma_{P u}^{2}} \cdot \frac{1}{(\rho L)_{\text {Fuel }}^{2}} \cdot\left(\frac{1}{S_{\text {elastic }}^{\prime}}+\frac{1}{S_{P u}^{\prime}+S_{\text {elassic }}^{\prime}}\right) \\
& \Rightarrow t_{f} \approx \frac{1}{n^{2} p^{2}} \cdot\left(\frac{M_{\text {Fuel }}}{N_{A}}\right)^{2} \cdot \frac{1}{\sigma_{P u}^{2}} \cdot \frac{1}{(\rho L)_{\text {Fuel }}^{2}} \\
& \otimes\left(\frac{M_{P_{u}}}{\alpha \varepsilon(\rho l)_{P u} N_{A} \sigma_{\text {elastic }} \Gamma \Phi_{o f f}^{\prime}(n)}+\frac{M_{P u}}{\alpha \varepsilon(\rho l)_{P u} N_{A}\left(\sigma_{P u}+\sigma_{\text {elastic }} \Gamma\right) \Gamma \Phi_{o n}^{\prime}(n)}\right) \\
& \rightarrow t_{f} \approx \frac{1}{n^{2} p^{2}} \cdot \frac{M_{F u l}^{2} M_{P_{u}}}{N_{A}^{3}} \cdot \frac{1}{\sigma_{P u}^{2}} \cdot \frac{1}{\sigma_{\text {elassic }}} \cdot \frac{1}{\Gamma\left(\alpha_{0} \varepsilon\right)_{P u}} \cdot \frac{1}{\Phi_{P_{u}}} \cdot g\left((\rho l)_{P u},(\rho L)_{F u e l} ; P u\right)
\end{aligned}
$$

Where $g\left((\rho l)_{P u}(\rho L)_{F u l} ; U\right)$ is a function of areal thickness of the plutonium witness foil and the fuel, where we have plugged in similar expressions as in Eq. 18 with $\mu_{\mathrm{Pu}-}$ $\operatorname{NRF}(\mathrm{n})$ and $\mu_{\mathrm{A}-\mathrm{Fuel}}$ is the nuclear and atomic attenuation through the fuel, respectively, and $\mu_{\mathrm{Pu}-\mathrm{NRF}}$ and $\mu_{\mathrm{A}-\mathrm{WF}}$ is the nuclear and atomic attenuation through the witness foil, respectively. We have assumed that the on- and off-channels are sufficiently close to approximate equality with the detector efficiency, atomic attenuation $\left(\mu_{\mathrm{A}}\right)$, elastic cross-sections ( $\sigma_{\text {elastic }}$ ), and flux. We have also assumed a large enough flux to overcome the natural radioactive background from the witness foil.

We now proceed by differentiating $t_{\mathrm{f}}$ with respect to the witness foil thickness and noting that $t_{\mathrm{f}}$ can never be zero. The issue we face here that we haven't faced with the $r$-method is that the witness foil thickness is a function of the fuel thickness. This makes sense because the upstream Pu witness foil attenuates NRF photons as well as the Pu nuclei in the fuel. Both create a notch in the flux and hamper the downstream NRF signal. Therefore, they must be coupled. We plot $t_{\mathrm{f}}$ per source photon [1/eV/sec] in Fig. 15 for 239Pu and 240Pu as a function of witness foil thickness. Figure 15 shows two sets of solid lines for $239 \mathrm{Pu}$ and $240 \mathrm{Pu}$, which represent different fuel thicknesses, namely the thickness of a single rod and the thickness of a full PWR assembly. The minimum for 239Pu occurs at

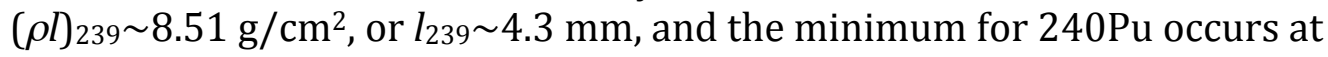
$(\rho)_{240} \sim 4.15 \mathrm{~g} / \mathrm{cm}^{2}$, or $l_{240} \sim 2.0 \mathrm{~mm}$. Comparison of the $\mathrm{LO}$ and LO+NLO approach for large NRF cross-sections we note a considerable difference as the witness foil thickness increases. We expect this because we are increasing the upstream witness foil and depleting the resonant photon that is necessary for the NRF counts downstream. This is especially true for large NRF cross-sections.

We see in Fig. 15 that for small fuel thicknesses 240Pu assay has a shorter time. This is expected because $240 \mathrm{Pu}$ has a higher cross-section than $239 \mathrm{Pu}$ but is somewhat offset with a lower concentration relative to $239 \mathrm{Pu}$. Assay time for full 
assemblies are longer as is expected because of the amount of material that must be traversed.

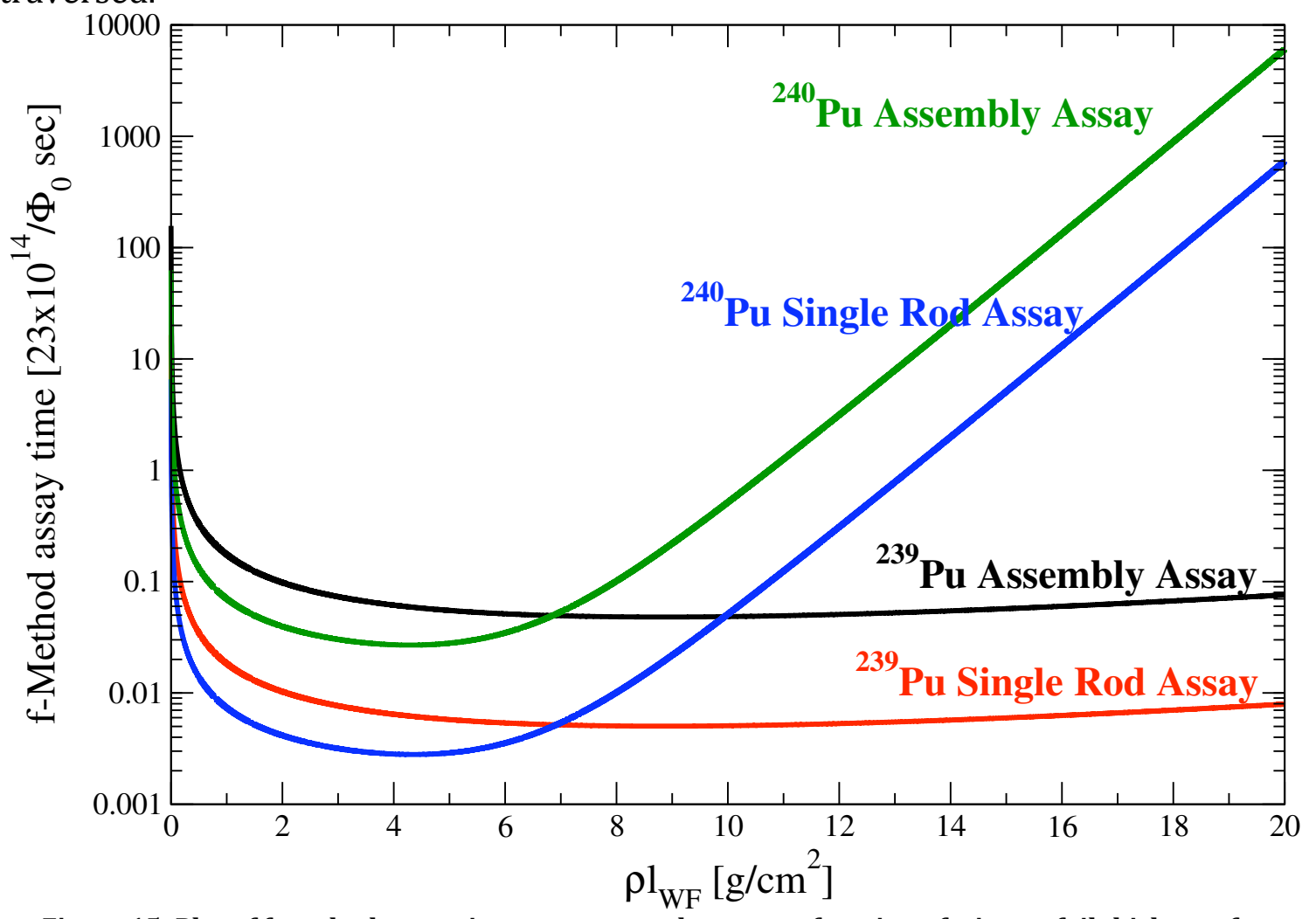

Figure 15: Plot of f-method assay time per source photon as a function of witness foil thickness for $239 \mathrm{Pu}$ and $240 \mathrm{Pu}$. The curves represent LO+NLO calculations. Plotted are two curves of each Pu isotope one for a single rod and one for the thickness of a PWR assembly. Pu concentration is $1 \%$. Precision is $1 \%$.

With the optimum values for the witness foils, we can plot the LO+NLO terms for the $f$-method assay times for 239Pu (black) and 240Pu (red), see Fig. 16. In Fig. 16 we have denoted the thicknesses for a single fuel rod and a full PWR assembly. There is a factor of 2 that separates both curves near the single fuel rod, which is consistent with Fig. 14. Note that the minimum for assaying spent fuel with the $f$ method is located around $45 \mathrm{~g} / \mathrm{cm}^{2}$, which is approximately a factor of 2 greater than the minimum for the $r$-method, see Fig. 13.

Figure 16 implies that if we were to use a quasi-monoenergetic source to assay an entire 17x17 PWR fuel assembly in one day we would need a source with about $10^{13}$ photons/eV/sec. You may be able to do a factor of 5 better if the assembly was broken down into subassemblies that are 4 pins thick. That is to say, use the thickness associated with the minimum (optimum time) of Fig. 16.

In Johnson et al., see [ND2010], measurement results were presented that indicated that NRF resonances in 239Pu might exist beyond the known states given in [Ber2008]. With the preliminary results from recent Pu measurements given in Tab. 3, it seems that these may indeed exist. Johnson et al., [ND2010], also indicated that these resonances, if they do exist, may have integrated cross-sections as large as $\sim 30 \mathrm{eV} \mathrm{b}$. A state this strong in $239 \mathrm{Pu}$ would not be a surprise since a similar 
strength cross-section has been found in 235U, see [Ber2008]. If we assume that a state such as this does exist and we choose a precision value of $3 \%$ and use a source that is $\sim 10^{6}$ photons/eV/sec (in development [Bar2009, Bar2011]), our calculations show that we could perform assay on a single pin in about $\sim 10^{3}$ seconds (i.e. $\sim 20$ minutes).

The statement above points to the importance of performing NRF measurements to find strong resonances. However, we are that mercy of nature and if there are no stronger resonances than what has already been reported, [Ber2008] and this work, the burden for faster count times falls upon the source.

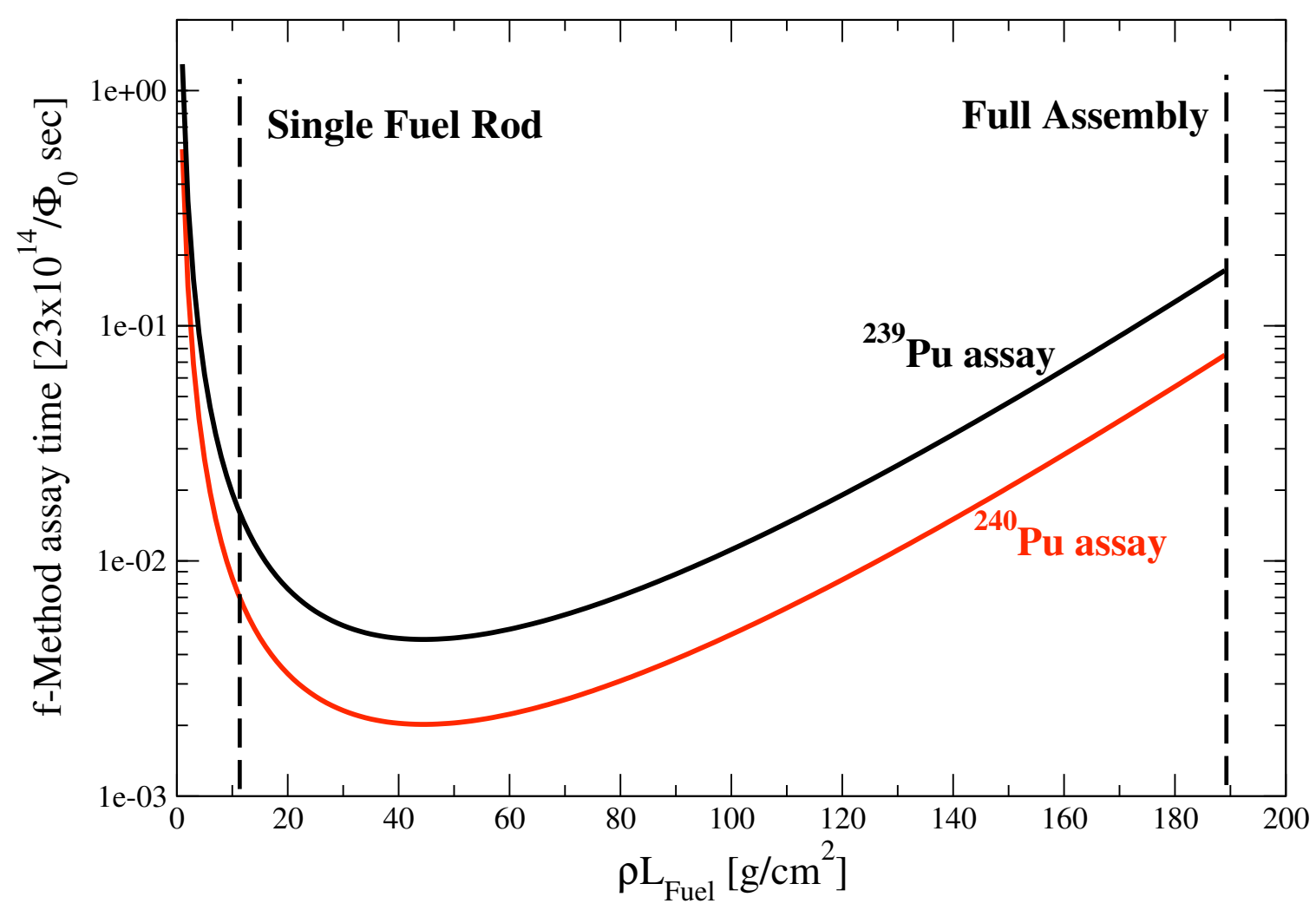

Figure 16: Assay time per source photon for 239Pu (black) and 240Pu (red). Also shown are the thicknesses of a single fuel rod and a PWR assembly. Pu concentration is $1 \%$. Precision is $1 \%$.

If we changed the scenario to assay $235 \mathrm{U}$ instead of Pu at an IE of $~ 3 \%$ and to a precision value of $3 \%$ and used the strongest NRF state at $1.733 \mathrm{MeV}$ [Ber2008] and use a source that is $\sim 10^{6}$ photons/eV/sec (in development at LLNL [Bar2009, Bar2011]), we could perform assay on a single pin in about $10^{2}$ seconds (i.e. $\sim 5$ minutes).

We now wish to compare the $r$-method and $f$-method. To do this we simply divide Fig. 13 by Fig. 16 and we get Fig. 17. Figure 17 indicates that for smaller fuel thicknesses, assaying 239Pu in the $r$-method is more advantageous. However, assaying $240 \mathrm{Pu}$ for any fuel thickness, even in small quantity is much more advantageous in the $f$-method. 


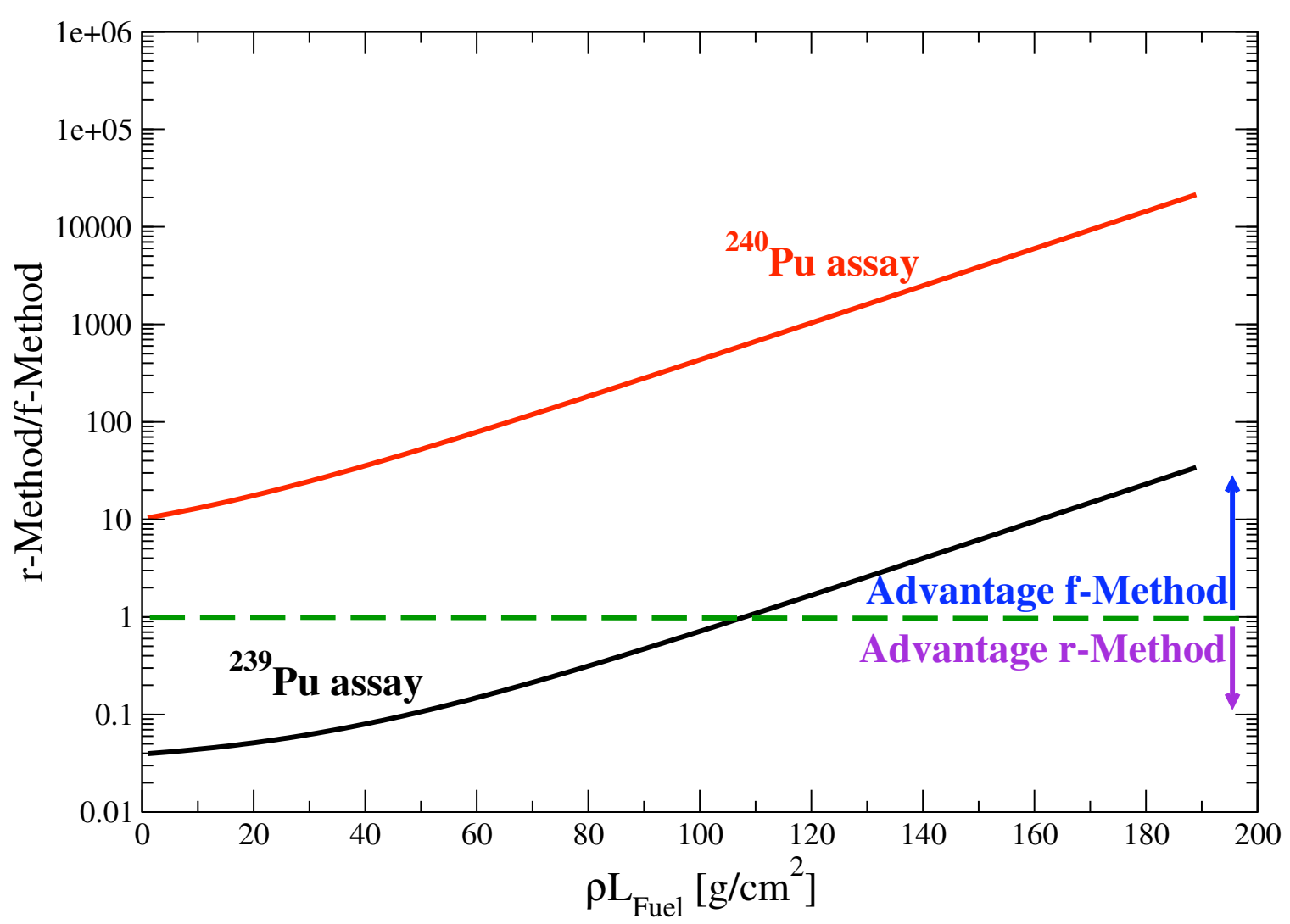

Figure 17: A comparison plot of the r-method to the f-method for 239Pu (black) and 240Pu (red). Denoted in the figure is a delineation of where one method is advantageous over the other. $\mathrm{Pu}$ concentration is $1 \%$. Precision is $1 \%$.

As a final discussion point we ask ourselves, could we do better with a bremsstrahlung source? If we use Eq. 1 as a crude estimator of how bremsstrahlung would perform (neglecting multiple scattering events that bremsstrahlung photons undergo and increased backgrounds from photo-fission in the Pu witness foil), and assume the same number of photons on resonance, we would find the constraint on our $\mathrm{Pb}$ absorber to be $2 \mathrm{~cm}$ thicker and would amount to count times 3 times longer than a quasi-monoenergetic source about $250 \mathrm{keV}$ wide (see Sec. NRF Methods), or a factor of 15 times longer in all.

What methods become more advantageous using quasi-monoenergetic sources versus bremsstrahlung? The answer is, it depends. It depends on the NRF strengths, end-point energy, multiple scattering effects, detector filters, etc... For now we answer that question simply by taking the ratio of the $r$-method and the $f$ method and factor in Eq. 21 to get:

$$
\frac{t_{r}}{t_{f}}=\frac{M_{U}}{M_{P u}} \cdot \frac{(\varepsilon)_{P u}}{(\varepsilon)_{U}} \cdot\left(\frac{\Gamma \sigma_{\text {elastic }}}{\sigma_{U}}\right)^{2} \cdot \underset{\text { Equation 23 }}{\left(2+\frac{\sigma_{U}}{\Gamma \sigma_{\text {elastic }}}\right)} \cdot F\left((\rho l)_{U},(\rho l)_{P u},(\rho L)_{F \text { Fuel }}\right)
$$

where the $F\left((\rho l)_{U,}(\rho l)_{P u}(\rho L)_{F u e l}\right)$ is a function of $f(\ldots)$ and $g(\ldots)$ are taken from Eqs. 17 and 22, respectively. Brems and QM denote the choice of bremsstrahlung or quasi-monoenergetic sources, respectively. Note that if we choose $Q M$, we get 
exactly Fig. 17. Also note that the detector absorbers are subscripted with Brems and $Q M$ to distinguish the higher attenuation one must use for bremsstrahlung (see Sec. NRF-Based Assay Methods). The difficulty with bremsstrahlung is that the backgrounds from multiple scattering will play a role, although it is unclear how large a role.

For either approach, the $r$-method or the $f$-method (Figs 13 and 16, respectively), the time to assay is inversely proportional to the flux (see Eqs. 20 and 22). Our calculations indicate that to scan an entire $17 \times 17$ PWR fuel assembly to a $1 \%$ precision in one day, a flux of about $\sim 10^{12}$ photons $/ \mathrm{eV} / \mathrm{sec}$ would be required. Detailed calculations show that a Joule-class Laser Compton backscatter source (LCS) may achieve $\sim 10^{18}$ photons/eV/sec [Har2005]. LLNL is developing LCS sources to study this unexplored brightness-energy region. The near goal ( $\sim 3-5$ years) for the first generation of developing sources at LLNL are $\sim 10^{6}$ photons/eV/sec [Bar2009, Bar2011]. If this goal were achieved, that would imply a substantial increase (about 4 orders of magnitude) in flux from the current bright source (i.e. HIGS) to LLNL's design. Mega-ray sources are very narrow bandwidth and may not be useful for the $r$-method, but could fair well for the $f$-method. HIGS researchers are also studying ways to increase the brightness of storage ring-based LCSs by orders of magnitude $\sim 10^{9}$ photons $/ \mathrm{eV} / \mathrm{sec}$ [Yu2011]. Off-the-shelf, $9 \mathrm{MeV}$, $50 \mathrm{~mA} \mathrm{CW}$, bremsstrahlung machines put out $\sim 5 \times 10^{8}$ photons $/ \mathrm{eV} / \mathrm{sec}$, but recall from our calculations above we lose about an order of magnitude in counting time because of the extra absorber thickness required for bremsstrahlung sources.

Certainly the inertia is driving towards brighter sources, which puts NRFbased assay on the horizon.

\section{Summary:}

We have studied the efficacy of using NRF for assay of spent nuclear fuel to determine Pu content with quasi-monoenergetic sources. We developed two methods ( $r$-method, $f$-method) to precisely determine the Pu content within a fuel $\mathrm{rod} / \mathrm{pin}$. In the $r$-method, precise determination of the ratio of $\mathrm{Pu}$ to $238 \mathrm{U}$ can be made using reflection or transmission detection modes. We have calculated the time required to measure the ratio of $\mathrm{Pu}$ isotopes to $\mathrm{U}$ isotopes to within $1 \%$ precision.

In the $f$-method, we have outlined a way to measure the mass of Pu that is nearly free of systematic uncertainties. We have determined the amount of time to measure the Pu mass to within 1\% precision. We have shown that for both methods advances in source brightness will directly improve the count times. We have also shown that $\mathrm{Pu}$ mass can be determined in the $f$-method just as quickly in a fuel assembly as a single fuel pin.

NRF-based assay measures the microscopic values of isotopes. Most, if not all other NDA methods only measure bulk values to determine Pu mass within a broad averaging distribution. No other NDA method (in situ or proposed) allows for such an isotopic determination. 


\section{References:}

- [And2010] S. Anderson. et al., LLNL LDRD 10-ERD-026 (2010).

- [Bar2009] C.P.J. Barty, et al., LLNL LDRD 09-SI-004 (2009).

- [Bar2011] C.P.J. Barty, Staff Scientist at LLNL, Private Communication, (2011)

- [Ber2005] William Bertozzi and Robert J. Ledoux, NIM B241, 820 (2005),

- [Ber2007] William Bertozzi, et al., NIM B261, 331 (2007).

- [Ber2008] W. Bertozzi, et al., Phys. Rev. C 78, 041601 (2008).

- [Cau1981] Y. Cauchois et al., J. Phys. G: Nucl. Phys. 71539 (1981)

- [Dys1959] N.A. Dyson, Proc. Phys. Soc. 73924 (1959)

- [GEANT4] GEANT4 Collaboration, Nucl. Inst. \& Meth. A506, 250 (2003).

- [Gos1990] T.B. Gosnell, UCRL 102805 (1990)

- [Hag2009] C.A. Hagmann et al., J. Appl. Phys. 99, 084901 (2009).

- [Haj2009] R. Hajima et al., Nucl. Inst. \& Meth. A (2009)

- [Ham2011] Y. Ham, Staff Scientist at LLNL, Private Communication, (2011)

- [Har2005] F.V. Hartemann et al., Phys. Rev. ST, AB, 8100702 (2005)

- [Hei1988] R.D. Heil et al., Nucl. Inst. \& Meth. A476, 39 (1988).

- [Her1986] O.W. Hermann and C.W. Alexander, ORNL/CSD/TM-205 (1986)

- [Joh2010] M.S. Johnson et al., Conf. Proc. of the 51 ${ }^{\text {st }}$ Ann. Mtg of the INMM, CD-ROM (2010)

- [Joh2011] M.S. Johnson et al., NIM B. \{Submitted for Publication\} (2011)

- [Kur1994] Kuriba, M. and Nemoto, N., IEEE Trans. Nucl. Sci., 331 (1994).

- [Leb2001] Lebrun, A., Symposium on International Safeguards (2001).

- [ND2010] M.S. Johnson et al., Conf. Proc. of Nuclear Data 2010 \{In Press\} (2010)

- [Pie2002] N. Pietralla et al., Phys. Rev. Lett. 88, 012502 (2002)

- [PrD2006] J.A. Pruet and D. P. McNabb, UCRL-TR-219071 (2006).

- [Pru2006] J.A. Pruet, et al., J. Appl. Phys. 99, 123102 (2006).

- [Rin1988] Rinard, P.M. and Bosler, G.E., LA-11096-MS (1988).

- [Taj2011] Prof. Toshiki Tajima, Research Prof. at The Munich-Centre for Advanced Photonics, Private Communication, (2011)

- [Tob2008] S.J. Tobin et al., Conf. Proc. for 49th Ann. Mtg. of the INMM (2008).

- [Vog2010] R. Vogt, Staff Scientist at LLNL, Private Communication, (2010)

- [Yin2011] Ying Wu, Assoc. Prof., Duke, Chief Scientist at HIGS, Private Communication, (2011) 